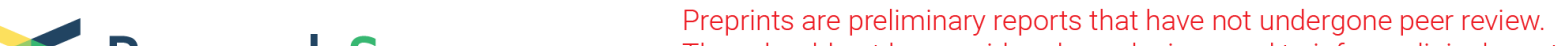 Research Square
or referenced by the media as validated information.
}

\section{A phylogenetically-restricted essential cell cycle progression factor in the human pathogen Candida albicans}

\section{Priya Jaitly}

Jawaharlal Nehru Centre for Advanced Scientific Research

\section{Melanie Legrand}

Institut Pasteur

\section{Abhijit Das}

Jawaharlal Nehru Centre for Advanced Scientific Research https://orcid.org/0000-0002-4891-5037

\section{Tejas Patel}

Jawaharlal Nehru Centre for Advanced Scientific Research https://orcid.org/0000-0002-4623-3657

\section{Murielle Chauvel}

Institut Pasteur

\section{Christophe d'Enfert}

Institut Pasteur https://orcid.org/0000-0002-6235-3886

\section{Kaustuv Sanyal ( $\nabla$ sanyal@jncasr.ac.in )}

Jawaharlal Nehru Centre for Advanced Scientific Research https://orcid.org/0000-0002-6611-4073

\section{Article}

Keywords: C. albicans, chromosomal stability, CSA6

Posted Date: October 26th, 2021

DOl: https://doi.org/10.21203/rs.3.rs-966555/v1

License: (c) (i) This work is licensed under a Creative Commons Attribution 4.0 International License. Read Full License

Version of Record: A version of this preprint was published at Nature Communications on July 23rd, 2022. See the published version at https://doi.org/10.1038/s41467-022-31980-3. 


8

\title{
A phylogenetically-restricted essential cell cycle progression factor in the human pathogen Candida albicans
}

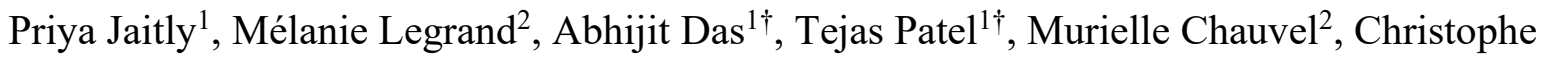 \\ $\mathrm{d}^{\prime}$ Enfert $^{2 *}$ and Kaustuv Sanyal ${ }^{1,3^{*}}$
}

${ }^{1}$ Molecular Mycology Laboratory, Molecular Biology and Genetics Unit, Jawaharlal Nehru Centre for Advanced Scientific Research, Bangalore, India.

${ }^{2}$ Institut Pasteur, Université de Paris, INRAE, USC2019, Unité Biologie et Pathogénicité

Fongiques, F-75015 Paris, France.

${ }^{3}$ Osaka University, Suita, Osaka, Japan.

* Corresponding authors. Email: christophe.denfert@pasteur.fr, sanyal@jncasr.ac.in

$\uparrow$ These authors contributed equally to this work.

(8)

(1)

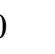

1

2

23

4

5

6

7




\section{Abstract}

Chromosomal instability in fungal pathogens caused by cell division errors is associated with antifungal drug resistance. To identify mechanisms underlying such instability and to uncover new potential antifungal targets, we conducted an overexpression screen monitoring chromosomal stability in the human fungal pathogen Candida albicans. Analysis of $~ 1000$ genes uncovered six chromosomal stability ( $C S A)$ genes, five of which are related to cell division genes in other organisms. The sixth gene, CSA6, is selectively present in the CUG-Ser clade species that includes $C$. albicans and other human fungal pathogens. The protein encoded by CSA6 localizes to the spindle pole bodies, is required for exit from mitosis, and induces a checkpoint-dependent metaphase arrest upon overexpression. Together, Csa6 defines an essential CUG-Ser fungal clade-specific cell cycle progression factor, highlighting the existence of phylogeneticallyrestricted cell division genes which may serve as potential unique therapeutic targets.

\section{Teaser}

Csa6 is essential for mitotic progression and mitotic exit in the human fungal pathogen Candida albicans.

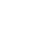

3

4

5

5

(1)

(

\section{Teaser} atbicans. 
Cell division is a fundamental aspect of all living organisms, required to support growth, reproduction and replenishment of dead or damaged cells. The primary objective of cell division is to ensure genome stability by preserving and transferring the genetic material with high-fidelity into progeny. Genome stability is achieved by proper execution of key cell cycle events such as chromosome duplication at the $\mathrm{S}$ phase followed by equal segregation of the duplicated chromosomes at the $\mathrm{M}$ phase. In addition, various cell cycle checkpoints monitor the integrity and fidelity of cell cycle events in response to an error or any damage until rectified or repaired. Failure of any of the error-correcting mechanisms can introduce genetic alterations, causing genomic instability in progeny. Genome instability can occur as a consequence of either point mutations, insertions or deletions of bases in specific genes and/or gain, loss or rearrangements of chromosomes, collectively referred to as chromosome instability (CIN) ${ }^{1}$. CIN has been intimately associated with aneuploidy ${ }^{2}$ and is one of the potential drivers of human genetic and neurodegenerative disorders ${ }^{3,4}$, aging ${ }^{5}$ and several cancers ${ }^{6}$. While considered harmful for a cell or an organism, CIN may also contribute to generating variations and help in driving evolution, especially in unicellular primarily asexual eukaryotes ${ }^{7,8}$.

The current understanding of the mechanisms underlying genome stability has evolved through studies in a range of biological systems from unicellular yeasts to more complex metazoa including humans. These studies highlighted concerted actions of genes involved in (a) highfidelity DNA replication and DNA damage repair, (b) efficient segregation of chromosomes and (c) error-correcting cellular surveillance machinery ${ }^{9,10}$ in maintenance and inheritance of a stable genome. In recent years, large-scale screenings of loss-of-function ${ }^{11}$, reduction-of-function ${ }^{12}$ and overexpression ${ }^{13,14,15,16}$ mutant collections in the budding yeast Saccharomyces cerevisiae have appended the list of genome stability-regulators by identifying uncharacterized proteins as well as known proteins with functions in other cellular processes. However, considering the vast diversity of the chromosomal segregation mechanisms in eukaryotes, it is conceivable that many genes involved in genome maintenance are yet to be discovered and require additional screens in a wide range of organisms for their identification. While perturbation of a candidate gene's function to decipher its role in a cellular pathway has been a classical strategy in biological research, screening of strain collections aids in uncovering molecular players and cellular pathways in an unbiased manner. 
The ascomycetous yeast Candida albicans is emerging as an attractive unicellular model for studying eukaryotic genome biology ${ }^{17}$. C. albicans, a commensal of both the gastrointestinal and genital tracts, remains the most frequently isolated fungal species worldwide from the patients diagnosed with candidemia or other nosocomial Candida infections ${ }^{18,19}$. The diploid genome of C. albicans shows remarkable plasticity in terms of ploidy, single nucleotide polymorphism (SNP), loss of heterozygosity (LOH), copy number variations, and CIN events ${ }^{17,20}$. Although LOH can be detected on all the chromosomes of $C$. albicans, the presence of recessive lethal or deleterious alleles on some haplotypes ${ }^{17}$, prevents one of the haplotypes or even a part of it from existing in the homozygous state. In particular, this homozygous bias has been observed for chromosomes R (ChR), 2 (Ch2), 4 (Ch4), 6 (Ch6) and 7 (Ch7) ${ }^{21,22}$. LOH and aneuploidy-driven CIN has serious phenotypic consequences in C. albicans such as conferring resistance to antifungals $23,24,25,26,27,28$ or help C. albicans adapt to different host niches $29,30,31$. Whether genome plasticity is contributing to the success of $C$. albicans as a commensal or/and a dreaded pathogen of humans, remains an enigma ${ }^{17}$. Nevertheless, with increasing instances of Candida infections and emerging antifungal resistance, there is an immediate need to identify novel fungus-specific molecular targets that may aid the development of antifungal therapies. In addition, the remarkable ability of $C$. albicans to tolerate CIN in the form of whole chromosome loss, isochromosome formation, chromosome truncation, or mitotic crossing-over ${ }^{17,20,32}$ raises intriguing questions on the functioning of genome stability-regulators in this fungal pathogen.

Of utmost importance to maintain genomic integrity, is the efficient and error-free segregation of the replicated chromosomes. In most eukaryotes including C. albicans, the assembly of a macromolecular protein complex, called the kinetochore (KT), on CENP-A (Cse4 in budding yeasts) containing centromeric chromatin mediates chromosome segregation during mitosis 33,34 , 35. The KT acts as a bridge between a chromosome and the connecting microtubules (MTs), emanating from the spindle pole bodies (SPBs), the functional homolog of centrosomes in mammals ${ }^{36}$. The subsequent attachment of sister KTs to opposite spindle poles then promotes the formation of a bipolar mitotic spindle that drives the separation of the duplicated chromosomes during anaphase ${ }^{37}$, after which cells exit mitosis and undergo cytokinesis ${ }^{38,39,40}$. In C. albicans, KT proteins remain clustered throughout the cell cycle and are shown to be essential for viability and mitotic progression $33,41,42$. In addition, genes involved in homologous recombination, such as MRE11 and RAD50, and DNA damage checkpoint pathway, including MEC1, RAD53 and DUN1, are required to prevent genome instability in C. albicans $43,44,45$. Strikingly, aberrant expression of proteins involved in DNA damage response or cell division triggers morphological 
transition to a unique polarized, filamentous growth in C. albicans ${ }^{17}$. A recent screen, using a collection of 124 over-expression strains, has identified three additional genes, namely, CDC20, $B I M 1$, and $R A D 51$, with a role in genome maintenance as indicated by increased $\mathrm{LOH}-$ driven CIN upon overexpression in C. albicans ${ }^{46}$. Currently, only a minor fraction of the C. albicans gene armamentarium has been evaluated for their roles in genome stability. Systematic approaches are thus needed to exhaustively define the drivers of $C$. albicans genome maintenance and outline species-specific processes as well as commonalities with other eukaryotes.

Here, we describe a large-scale screen aimed at identifying regulators of genome stability in a clinically relevant fungal model system. Our screen, involving $20 \%$ of the C. albicans ORFeome, has identified Csa6, a yet unknown player of genome stability, as a critical regulator of cell cycle progression in C. albicans. Overall, this is the first-ever report of such a screen at this scale in C. albicans and provides a framework for identifying regulators of eukaryotic genome stability, some of which may serve as new targets for therapeutic interventions of fungal infections.

\section{Results}

\section{A reporter system for monitoring chromosome stability in $C$. albicans}

To understand the molecular mechanisms underlying genome instability in a fungal pathogen, we developed a reporter system in C. albicans in which whole chromosome loss can be distinguished from other events such as break-induced replication, gene conversion, chromosome truncation or mitotic crossing over ${ }^{22,46}$. In our prior work, a loss-of-heterozygosity (LOH) reporter strain was developed for use in C. albicans ${ }^{22,46}$. In this strain GFP and BFP genes, linked to ARG4 and HIS1 auxotrophic markers, respectively, are integrated at the same intergenic locus on the left arms of chromosome 4A (Ch4A) and chromosome 4B (Ch4B), respectively (Fig. 1A, S1A) ${ }^{22}$. Consequently, cells express both GFP and BFP as analyzed by flow cytometry and are prototrophic for $A R G 4$ and HIS1 genes, unless a chromosome instability (CIN) event causes loss of one of the two loci (Fig. 1A, B ${ }^{22}$. To differentiate whole chromosome loss from other events that may lead to loss of one of the two reporter loci, we modified the $\mathrm{LOH}$ reporter strain by integrating a red fluorescent protein (RFP) reporter gene, associated with the hygromycin B (hyg B) resistance marker, on the right arm of Ch4B (Fig. 1A, S1A). The RFP reporter insertion is sufficiently distant from the BFP locus that loss of both BFP and RFP signal (and of their linked 
auxotrophic/resistance markers) is indicative of loss of Ch4B, rather than a localized event causing loss of the BFP-HIS1 reporter insertion (Fig. 1A, S1A). Notably, while loss of Ch4A cannot be tolerated due to the presence of recessive lethal alleles on $\mathrm{Ch} 4 \mathrm{~B}^{22}$, loss of Ch4B leads to formation of small colonies that mature into larger colonies following duplication of $\mathrm{Ch} 4 \mathrm{~A}^{46}$. Thus, the absence of both BFP-HIS1 and RFP-HYG B but continued presence of GFP-ARG4 in the modified reporter strain, which we named as chromosome stability (CSA) reporter, enables us monitor loss of $\mathrm{Ch} 4 \mathrm{~B}$ in a population. The fluorescence intensity profile of GFP, BFP and RFP in the CSA reporter was validated by flow cytometry (Fig. S1B). To functionally validate the CSA reporter system, we employed overexpression of $C D C 20$, a gene important for anaphase onset, activation of spindle assembly checkpoint and whose overexpression is known to cause whole chromosome loss in C. albicans ${ }^{46}$. We analyzed the BFP/GFP density plots in various control strains (Fig. S1C) and monitored the loss of BFP/GFP signal in cells overexpressing CDC20 $\left(C D C 20^{O E}\right)$ by flow cytometry. As reported earlier ${ }^{46}$, the $C D C 20^{O E}$ strain displayed a higher population of $\mathrm{BFP}^{+} \mathrm{GFP}^{-}$and $\mathrm{BFP}^{-} \mathrm{GFP}^{+}$cells as compared to the empty vector (EV) control indicating increased $\mathrm{CIN}$ in the $C D C 20^{O E}$ mutant (Fig. S1D, E). Next, we isolated BFP-GFP ${ }^{+}$cells of EV and $C D C 20^{O E}$ using flow cytometry and plated them for subsequent analysis of auxotrophic/resistance markers (Fig. S1F). As noted above, upon incubation of the sorted BFP$\mathrm{GFP}^{+}$cells, we observed the appearance of both small and large colonies (Fig. S1F). Small colonies have been previously shown to be the result of loss of $\mathrm{Chr} 4 \mathrm{~B}$ homolog and are predicted to be a consequence of Ch4A monosomy, eventually yielding large colonies upon reduplication of $\mathrm{Ch} 4 \mathrm{~A}^{46}$. We, therefore, performed the marker analysis on large colonies and found that $85 \%$ of the $\mathrm{BFP}^{-} \mathrm{GFP}^{+}$derived colonies of $C D C 20^{O E}$ mutant concomitantly lost both HISI and HYG B but retained ARG4 (Fig. S1G) suggesting the loss of Ch4B homolog; flow cytometry analysis further confirmed the loss of BFP and RFP signals in these colonies. The remaining $15 \%$ of colonies retained GFP-ARG4 and RFP-HYG B but not BFP-HIS1 (Fig. S1G) indicating that more localized events including gene conversion, rather than whole chromosome loss, were responsible for loss of the BFP signals in these cells. The above data indicate that the CSA reporter system that we engineered enables precise monitoring of the whole chromosome loss event in a population and enables large-scale screening of this phenotype.

Medium-throughput screening of $C$. albicans overexpression strains identifies regulators of genome stability 
Systematic gene overexpression is an attractive approach for performing large-scale functional genomic analysis in C. albicans, a diploid ascomycete. Using a recently developed collection of C. albicans inducible overexpression plasmids (Chauvel et al., manuscript in preparation) and the CSA reporter strain described above, we generated a library of 1067 C. albicans inducible overexpression strains. Each of these strains, carrying a unique ORF under control of the $P_{T E T}$ promoter, could be induced for overexpression after anhydrotetracycline (Atc) or doxycycline (Dox) addition (Fig. 1C) ${ }^{46,47}$. To identify regulators of genome stability, we carried out a primary screen with these 1067 overexpression strains by individually analyzing them for the loss of BFP/GFP signals by flow cytometry (Fig. 1C, S2A, Dataset 1). Our primary screening identified 23 candidate genes (out of 1067) whose overexpression resulted in $\geq 2$-fold increase in the $\mathrm{BFP}^{+} \mathrm{GFP}^{-}$and $\mathrm{BFP}^{-} \mathrm{GFP}^{+}$population relative to the EV (Table S1, S2). Next, we carried out a secondary screen with these 23 overexpression strains to revalidate the loss of BFP/GFP markers by flow cytometry (Fig. 1C, S2B). As genotoxic stress is intimately linked with polarized growth in C. albicans ${ }^{17}$, 48 , we microscopically examined the overexpression strains exhibiting higher instability at the BFP/GFP locus during secondary screening for any morphological transition (Fig. 1C, S2B). While overexpression of 17 genes (out of 23) could not reproduce the BFP/GFP loss phenotype, overexpression of the six genes resulted in $\geq 2$-fold increase in the $\mathrm{BFP}^{+} \mathrm{GFP}^{-}$or $\mathrm{BFP}^{-} \mathrm{GFP}^{+}$population as compared to the $\mathrm{EV}$, with three genes (out of 6 ) inducing polarized growth upon overexpression (Fig. S3A, B). These six genes, which we referred to as CSA genes, include CSA1 (CLB4), CSA2 (ASE1), CSA3 (KIP2), CSA4 (MCM7), CSA5 (BFA1) and CSA6 coded by ORF19.1447 of unknown function (Fig. 1D).

\section{Molecular mechanisms underlying $\mathrm{CIN}$ in $C S A$ overexpression mutants}

Out of the six CSA genes, overexpression of three genes, namely, $C S A 1^{C L B 4}, C S A 2^{A S E 1}$ and $C S A 3^{K I P 2}$ caused little or no change in the morphology of C. albicans (Fig. S3A), but triggered $\mathrm{CIN}$ at the $\mathrm{BFP} / \mathrm{GFP}$ locus, indicated by an expansion of the $\mathrm{BFP}^{+} \mathrm{GFP}^{-}$and $\mathrm{BFP}^{-} \mathrm{GFP}^{+}$population in the flow cytometry density plots (Fig. S3B, C). To further dissect the molecular mechanisms leading to the loss of $\mathrm{BFP} / \mathrm{GFP}$ signals in these mutants, we sorted $\mathrm{BFP}^{-} \mathrm{GFP}^{+}$cells of these mutants and plated them for GFP-ARG4,BFP-HIS1 and RFP-HYG B analysis, as described previously for the $C D C 20^{O E}$ mutant. We observed that a majority of the large $\mathrm{BFP}^{-} \mathrm{GFP}^{+}$derived colonies of $C S A 1^{C L B 4}, C S A 2^{A S E 1}$ and $C S A 3^{K I P 2}$ overexpression mutants lost BFP-HIS1 but retained $R F P-H Y G B$ and GFP-ARG4 (Fig. S3D), suggesting that localized genome instability events, 
rather than whole chromosome loss events, contributed to the high percentage of $\mathrm{BFP}^{-} \mathrm{GFP}^{+}$cells in these mutants.

Overexpression of the remaining three genes, namely $C S A 4^{M C M 7}, C S A 5^{B F A 1}$ and CSA6, drastically altered the morphology of the C. albicans cells by inducing polarized/filamentous growth (Fig. S3A). A connection between morphological switches and genotoxic stresses has been established in the polymorphic fungus $C$. albicans, wherein polarized growth is triggered in response to improper cell cycle regulation $41,42,48,49,50$. Flow cytometric analysis of cell cycle progression revealed that overexpression of $C S A 4^{M C M 7}, C S A 5^{B F A 1}$ and $C S A 6$ shifted cells towards the 4N DNA content (Fig. S3E). To further determine the cell cycle phase associated with the $4 \mathrm{~N}$ shift, we compared nuclear segregation patterns (Hoechst staining for DNA and CENP-A/Cse4 localization for KT) and spindle dynamics (separation of Tub4 foci) in these overexpression mutants with those of the EV control (Fig. S3F). Our results suggested the $4 \mathrm{~N}$ shift in $C S A 4^{M C M 7}$ and CSA6 overexpression mutants was a result of G2/M arrest, indicated by a high percentage of largebudded cells with unsegregated DNA mass and improperly separated SPBs (Fig. S3F). In contrast, the $4 \mathrm{~N}$ shift upon $C S A 5^{B F A}$ overexpression was a consequence of late anaphase/telophase arrest, shown by an increased number of large-budded cells with segregated nuclei and SPBs (Fig. S3F). Taken together, our results indicate that the polarized growth in each of $C S A 4^{M C M 7}$, $C S A 5^{B F A 1}$ and $C S A 6$ overexpression mutants is a probable outcome of improper cell cycle progression.

Two $C S A$ genes, namely $C S A 2^{A S E 1}$ and $C S A 5^{B F A 1}$, gave rise to similar overexpression phenotypes in both $S$. cerevisiae and C. albicans (Table 1). While phenotypes related to $C S A 4^{M C M 7}$ and $C S A 6$ overexpression in S. cerevisiae or other related organisms remained unreported, the overexpression phenotypes of the remaining CSA genes were along the lines of their roles in cell cycle functioning, as reported in S. cerevisiae (Table 1, Fig. 1D). Altogether, our results validated the role of CSA genes in regulating genome stability in $C$. albicans. While overexpression of either $C S A 1^{C L B 4}, C S A 2^{A S E 1}$ or $C S A 3^{K I P 2}$ induced CIN mostly through non-chromosomal loss events, the effect of overexpression of either $C S A 4^{M C M 7}, C S A 5^{B F A 1}$ or $C S A 6$ was so drastic that the C. albicans mutants were arrested at different cell cycle phases with G2/M equivalent DNA content $(4 \mathrm{~N})$ and thus were unable to complete the mitotic cell cycle.

\section{Csa6 is an SPB-localizing protein, present across a subset of CUG-Ser clade fungal species}


Among the genes identified in the screen, Csa6 was the only protein without any detectable homolog in S. cerevisiae (Fig. 1D). This intrigued us to examine its presence across various other fungi. Phylogenetic analysis using high confidence protein homology searches and synteny-based analysis indicated that Csa6 is exclusively present in a subset of fungal species belonging to the CUG-Ser clade (Fig. 2A). Strikingly, in all these species, Csa6 was predicted to have a central coiled-coil domain (Fig. 2B). Epitope tagging of Csa6 with a fluorescent marker (mCherry) localized it close to the $\mathrm{KT}$ throughout the cell cycle in C. albicans (Fig. 2C). In most unicellular fungi, often found proximal to the clustered KTs, are the SPB complexes ${ }^{33,35,51,52}$. Although neither the SPB structure nor its composition is well characterized in C. albicans, the majority of the SPB proteins exhibit high sequence and structural conservation from yeast to humans ${ }^{53}$. Hence, we re-examined Csa6 localization with two of the evolutionarily conserved SPB proteins, Tub4 and Spc110, in C. albicans ${ }^{53,54}$ (Fig. 2D, E). These results showed that Csa6 constitutively localizes to the SPBs, close to the KTs, in cycling yeast cells of C. albicans (Fig. 2D, E).

\section{Csa6, a previously uncharacterized protein, as a key regulator of mitotic progression in $C$.} albicans

While roles of Csa6 have not been investigated before, based on our findings thus far (Fig. S3E, F), we hypothesized that Csa6 plays an important function in cell cycle regulation and genome stability in C. albicans. We sought to identify the molecular pathways by which Csa6 performed its functions in C. albicans. We again made use of the inducible $\mathrm{P}_{T E T}$ promoter system to generate a CSA6 ${ }^{O E}$ strain (CaPJ176, $P_{T E T} C S A 6$ ) in the wild-type (SN148) background of C. albicans (Fig. 3A). Conditional overexpression of TAP-tagged Csa6 (CaPJ181, $P_{\text {TET }}$ CSA6-TAP), in presence of Atc, was confirmed by western blot analysis (Fig. 3B). The effect of $C S A 6^{O E}$ (CaPJ176, $P_{T E T} C S A$ ) on cell cycle functioning was then investigated by flow cytometric cell cycle analysis (Fig. 3C) and microscopic examination of the nuclear division (Fig. 3D). As observed previously (Fig. S3E, F), CSA6 ${ }^{O E}$ inhibited cell cycle progression in C. albicans by arresting cells in the G2/M phase, evidenced by the gradual accumulation of large-budded cells with unsegregated nuclei (Fig. 3D), possessing 4N DNA content (Fig. 3C). Some of these large-budded cells also underwent a morphological transition to an elongated bud or other complex multi-budded phenotypes (Fig. 3D), indicating cell cycle arrest-mediated morphological switching ${ }^{48}$ due to $C S A 6^{O E}$. Strikingly, continuous upregulation of Csa6 was toxic to the cells (Fig. S4A) as nuclei failed to segregate in this mutant (Fig. 3D). 
Nuclear segregation during mitosis is facilitated by the formation of the mitotic spindle and its dynamic interactions with chromosomes via KTs. Thus, we sought to examine both the KT integrity and the mitotic spindle morphology in the $C S A 6^{O E}$ mutants. In C. albicans, the structural stability of the KT is a determinant of CENP-A/Cse4 stability wherein depletion of any of the essential KT proteins results in delocalization and degradation of the CENP-A/Cse4 by ubiquitinmediated proteolysis ${ }^{50}$. Fluorescence microscopy and western blot analysis confirmed that Cse 4 was neither delocalized (Fig. S4B) nor degraded from centromeric chromatin (Fig. S4C) upon $C S A 6^{O E}$. Next, we analyzed the spindle integrity in $C S A 6^{O E}$ mutants by tagging Tub4 (SPB) and Tub1 (MTs) with fluorescent proteins. Fluorescence microscopy analysis revealed that a large proportion $(\sim 73 \%)$ of the large-budded cells formed an unconventional rudimentary mitotic spindle structure upon $C S A 6^{O E}$, wherein it had a dot-like appearance as opposed to an elongated bipolar rod-like spindle structure in EV or uninduced (-Atc) strains (Fig. 3E). This suggests that nuclear segregation defects in $C S A 6^{O E}$ mutant cells are an attribute of aberrant mitotic spindle formation that might have led to the mitotic arrest.

During mitosis, surveillance mechanisms, including spindle assembly checkpoint (SAC) ${ }^{55,56}$ and spindle positioning checkpoint (SPOC) ${ }^{57,58}$ operate to maintain genome stability by delaying the metaphase-anaphase transition in response to improper chromosome-spindle attachments and spindle misorientation, respectively. We posit that the G2/M cell cycle arrest due to $C S A 6^{O E}$ in $C$. albicans could be a result of either SAC or SPOC activation. Hence, we decided to inactivate SAC and SPOC, individually, in the $C S A 6^{O E}$ strain by deleting the key spindle checkpoint genes $M A D 2^{41}$ and $B U B 2^{48}$, respectively. SAC inactivation in $C S A 6^{O E}$ mutant cells (Fig. 4A) led to the emergence of unbudded cells with 2N DNA content (Fig. 4B, C), indicating a bypass of the G2/M arrest caused by $C S A 6^{O E}$. Consequently, we also observed a partial rescue of the growth defect in $C S A 6^{O E}$ mutant cells (Fig. S5A). Next, we sought to characterize the effect of SAC inactivation on the spindle integrity in $C S A 6^{O E}$ mutants. $C S A 6^{O E}$ resulted in the formation of an unconventional mitotic spindle (Fig. 3E) wherein it displayed a single focus of SPB (Tub4-GFP), colocalizing with a single focus of MTs (Tub1-mCherry). We speculated two possibilities that may lead to the single focus of Tub4: a) a defect in the process of SPB duplication or b) a delay in the separation of duplicated SPBs. Fluorescence microscopy analysis revealed that SAC inactivation in $C S A 6^{O E}$ mutant drastically increased the percentage of large-budded cells (from $\sim 30 \%$ to $\sim 68 \%$ ) with two separated SPB foci (Tub4-GFP) (Fig. S5B). These results ruled out the possibility of an unduplicated SPB in $C S A 6^{O E}$ mutant cells and hinted at the importance of cellular Csa6 levels for proper SPB separation and chromosome segregation in C. albicans. 
We next determined the effect of inactivating SPOC in the cells overexpressing Csa6. For this, we generated a $C S A 6^{O E}$ strain (CaPJ200) using the bub2 null mutant (CaPJ110) as the parent strain and monitored nuclear division following Hoechst staining. Strikingly, we did not observe a bypass of G2/M arrest in $C S A 6^{O E}$ mutant upon SPOC inactivation, indicated by a persistent population of large-budded cells with unsegregated nuclei (Fig. S5C). Altogether, our results demonstrate that overexpression of Csa6 leads to a Mad2-mediated metaphase arrest due to a malformed spindle in C. albicans.

\section{Csa6 regulates mitotic exit network and is essential for viability in $C$. albicans}

To further gain insights into the biological function of Csa6, we sought to generate a promoter shut-down mutant of $\operatorname{csa} 6\left(C S A 6^{P S D}\right)$. For this, we deleted one of its alleles and placed the remaining one under the control of the MET3 promoter ${ }^{59}$ which gets repressed in presence of methionine (Met/M) and cysteine (Cys/C) (Fig. 5A). Western blot analysis confirmed the depletion of TAP-tagged Csa 6 in $C S A 6^{P S D}$ mutant within $6 \mathrm{~h}$ of growth under repressive conditions (Fig. 5B). The inability of $C S A 6^{P S D}$ mutant to grow in non-permissive conditions confirmed the essentiality of Csa6 for viability in C. albicans (Fig. 5C). Subsequently, we analyzed the cell cycle profile (Fig. 5D) and nuclear division dynamics (Fig. 5E) in the CSA $6^{P S D}$ strain after a specific period of incubation in either permissive or non-permissive conditions. Strikingly, Csa6 depletion, as opposed to its overexpression, resulted in cell cycle arrest at the late anaphase/telophase stage, indicated by an increasing proportion of large-budded cells, possessing segregated nuclei and 4N DNA content (Fig. 5D, E). Additionally, we observed cells with more than two nuclei, elongated-budded cells and other complex phenotypes upon Csa6 depletion (Fig. 5E). While CENP-A/Cse4 remained localized to centromeres in $C S A \sigma^{P S D}$ mutant as revealed by the fluorescence microscopy (Fig. S6A), an increase in the cellular levels of Cse4 was observed in $C S A 6^{P S D}$ mutant by western blot analysis (Fig. S6B). The increase in Cse4 levels could be an outcome of Cse4 loading at anaphase in C. albicans ${ }^{60,61}$. Finally, we analyzed the integrity of the mitotic spindle, as mentioned previously, in $C S A 6^{P S D}$ mutant. We noticed the mean length of the anaphase mitotic spindle in Csa6-depleted cells was significantly higher $(\sim 11 \mu \mathrm{m})$ than that of the cells grown under permissive conditions ( $\sim 6 \mu \mathrm{m})$, indicating a spindle disassembly defect in $C S A 6^{P S D}$ mutant (Fig. 5F). 
A close link between anaphase arrest, hyper-elongated mitotic spindle and inactive mitotic exit network (MEN) have been established before ${ }^{40,62,63}$. Localized at the SPB, the MEN is a signaling cascade in $S$. cerevisiae that triggers cells to come out of mitosis and proceed to cytokinesis (Fig. 6A) ${ }^{64}$. We speculated the anaphase arrest in $C S A 6^{P S D}$ mutant could be a result of an inactive MEN signaling. To determine this, we sought to bypass the anaphase arrest associated with Csa6 depletion by overexpressing SOL1, the CDK inhibitor and Sic1 homolog in C. albicans 65 (Fig. 6B), using the inducible $\mathrm{P}_{T E T}$ system mentioned previously (Fig. 6C). The conditional overexpression of Protein A-tagged Sol1 upon addition of Atc was verified by western blot analysis (Fig. 6D). Strikingly, $S O L 1^{O E}$ in association with Csa6 depletion allowed cells to exit mitosis but not cytokinesis, as evidenced by the formation of chains of cells with $>4 \mathrm{~N}$ DNA content (Fig. 6E, F). To further examine the role of Csa6 in mitotic exit, we analyzed the localization of a MEN component, Tem1, a GTPase that is known to initiate MEN signaling 39, 66, 67, 68 . In C. albicans, Tem1 localizes to SPBs in a cell-cycle-regulated manner and is essential for viability ${ }^{39}$. Fluorescence microscopy revealed that while Tem1 is localized to both the SPBs in anaphase under permissive conditions (Fig. 6G) as observed earlier ${ }^{39}$, a high percentage of Csa6depleted cells ( $\sim 78 \%$ ) had Tem1 localized to only one of the two SPBs (Fig. 6G), suggesting an important role of Csa6 in regulating mitotic exit in C. albicans. Altogether, our results demonstrate that $\mathrm{Csa} 6$ is required for mitotic exit and thus essential for viability in C. albicans.

\section{Csa6 of Candida dubliniensis functionally complements Csa6 of C. albicans}

To further elucidate the intra-species function and localization of Csa6, we decided to ectopically express Csa6 of another CUG-Ser clade species, Candida dubliniensis (CdCsa6) in C. albicans. C. dubliniensis is a human pathogenic budding yeast that shares a high degree of DNA sequence homology with C. albicans, and possesses unique and different centromere DNA sequences on each of its eight chromosomes ${ }^{69,70}$. Upon protein sequence alignment, we found that CdCsa6 (ORF Cd36_16290) is 79\% identical to Csa6 of C. albicans (CaCsa6) (Fig. 7A). The ectopic expression of GFP-tagged CdCsa6 in C. albicans was carried out using the replicative plasmid pCdCsa6-GFP-ARS2 (Fig. 7B), which contains the autonomously replicating sequence (ARS) of C. albicans ${ }^{71}$. Although unstable when present in an episomal form, ARS plasmids, upon spontaneous integration into the genome, can propagate stably over generations ${ }^{72}$. Fluorescence microscopy of integrated pCdCsa6-GFP-ARS2 revealed that similar to CaCsa6, CdCsa6 localizes constitutively to the SPBs in C. albicans (Fig. 7C), further supporting Csa6's evolutionarily conserved role in regulating mitotic spindle and mitotic exit in C. albicans. We next asked if 
CdCsa6 can functionally complement CaCsa6. For this, we ectopically expressed CdCsa6 in $C S A \sigma^{P S D}$ strain. Strikingly, the ectopic expression of CdCsa6 rescued the growth defect associated with $C S A 6^{P S D}$ mutant under non-permissive conditions, indicating CdCsa6 can functionally complement CaCsa6 (Fig. 7D). This suggests functional conservation of Csa6 among related Candida species belonging to the CUG-Ser clade.

\section{Discussion}

In this study, we carried out an extensive screen to identify genes that contribute to genome stability in C. albicans by generating and analyzing a library of more than a thousand overexpression strains. Our screen identified six regulators of chromosome stability including Csa6, a protein of unknown function. Molecular dissection of Csa6 function revealed its importance in cell cycle progression at least in two critical stages, metaphase-anaphase transition and mitotic exit. We further demonstrated that Csa6 is constitutively localized to the SPBs, essential for viability, and alterations of its cellular level leads to cell cycle arrest in C. albicans. Finally, subcellular localization and complementation analysis revealed functional conservation of Csa 6 across the pathogenic Candida species.

The identification of two CSA genes, $C S A 2^{A S E I}$ and $C S A 5^{B F A 1}$, that were earlier reported as CIN genes ${ }^{13,14}$, further validates the power of the screening approach and the methods presented in this study. The respective overexpression phenotypes of these two genes in C. albicans were found to be similar to those in S. cerevisiae, suggesting that their functions might be conserved in these distantly related yeast species. In S. cerevisiae, Ase1 acts as an MT-bundling protein, required for spindle elongation and stabilization during anaphase ${ }^{73,74}$ (Fig. 8A). Hence, increased CIN upon ASE1 overexpression might be an outcome of premature spindle elongation and improper KT-microtubule attachments ${ }^{74,75}$. Bfa1, on the other hand, is a key component of the Bub2-Bfa1 complex, involved in SPOC activation ${ }^{57}$, and a negative regulator of mitotic exit ${ }^{76}$ (Fig. 8A). In S. cerevisiae, BFAl overexpression prevents Tem 1 from interacting with its downstream effector protein $\mathrm{Cdc} 15$, thus inhibiting MEN signaling and arresting cells at the anaphase ${ }^{77}$. In our screen, a B-type mitotic cyclin Clb4 (CSA1), and a kinesin-related motor protein Kip2 (CSA3) (Fig. 8A), were found to increase CIN upon overexpression, primarily via non-chromosomal loss events. C. albicans $\mathrm{Clb} 4$ acts as a negative regulator of polarized growth ${ }^{49}$ and is the functional homolog of S. cerevisiae $\mathrm{Clb}^{78}$, required for the entry into the S-phase ${ }^{79}$. Increased CIN upon $C_{S A 1}{ }^{C L B 4}$ overexpression, is thus consistent with its role in S-phase initiation. 
The function of Kip2, however, is yet to be characterized in C. albicans. In S. cerevisiae, Kip2

functions as an MT polymerase ${ }^{80}$, with its overexpression leading to hyperextended MTs and defects in SPB separation ${ }^{81}$. The associated CIN observed upon $C S A 3^{K I P 2}$ overexpression in $C$. albicans is in line with its function in nuclear segregation.

Mcm7, another CSA gene (CSA4) identified in this study, is a component of the highly conserved Mcm2-7 helicase complex, essential for eukaryotic DNA replication initiation and elongation ${ }^{82}$ (Fig. 8A). While Mcm7 depletion arrests cells at $\mathrm{S}$ phase ${ }^{83}$, the effect of $M C M 7$ overexpression on genomic integrity is comparatively less explored. Especially, several cancerous cells have been shown to overexpress Mcm $7^{84,85,86}$, with its elevated levels increasing the chances of relapse and local invasions ${ }^{84}$. In this study, we found that overexpression of $M C M 7$, in contrast to $\mathrm{Mcm} 7$ depletion, arrested cells at the G2/M stage. One possibility is increased Mcm7 levels interfered with DNA replication during the $\mathrm{S}$ phase, resulting in DNA damage or accumulation of singlestranded DNA, thus activating the $R A D 9$-dependent cell cycle arrest at the G2/M stage ${ }^{87,88}$. In a recent study from our laboratory, $\mathrm{Mcm} 7$ has been identified as a subunit of the kinetochore interactome in a basidiomycete yeast Cryptococcus neoformans ${ }^{89}$. Another subunit of the Mcm27 complex, Mcm2, is involved in regulating the stability of centromeric chromatin in C. albicans 61. Considering the growing evidence of the role of Mcm2-7 subunits beyond their canonical, well-established roles in DNA replication, the serendipitous identification of $\mathrm{Mcm} 7$ as a regulator of genome stability in our screen is striking.

We performed an in-depth analysis of Csa6, a novel regulator of cell cycle progression identified from our screen (Fig. 8B, C). Our results revealed that overexpression of CSA6 leads to an unconventional mitotic spindle formation and SAC-dependent G2/M cell cycle arrest (Fig. 8C) in C. albicans. While mad2 deletion indicated that SPB duplication and separation of duplicated SPBs is unperturbed in CSA6 overexpressing cells, what exactly triggered the activation of SAC in these cells remains to be determined. Recent studies on human cell lines have shown that failure in the timely separation of the centrosomes promotes defective chromosome-MT attachments and may lead to chromosome lagging if left uncorrected by the cellular surveillance machinery $90,91,92$. Along the same lines, we posit that a delay in SPB separation, mediated by overexpression of Csa6, leads to increased instances of improper chromosome-MT attachments, leading to SAC activation and an indefinite arrest at the metaphase stage. Future studies on the SPB structure-function and composition in C. albicans should reveal how Csa6 regulates SPB dynamics in this organism. 
In contrast to its overexpression, Csa6 depleted cells failed to exit mitosis and remained arrested at the late anaphase/telophase stage (Fig. 8C). We further linked the mitotic exit failure in Csa6 depleted cells with the defective localization of Tem1, an upstream MEN protein. While the hierarchy of MEN components, starting from the MEN scaffold Nud1, an SPB protein, to its ultimate effector Cdc14 is well established in S. cerevisiae ${ }^{64}$, the existence of a similar hierarchy in C. albicans needs to be investigated. In addition, several lines of evidence suggest that MEN in C. albicans may function differently from S. cerevisiae: (a) Unlike S. cerevisiae, C. albicans Cdc14 is non-essential for viability with its deletion affecting cell separation ${ }^{93}$. (b) Cdc14 is present in the nucleoplasm for the majority of the cell cycle in contrast to its nucleolar localization in S. cerevisiae ${ }^{93}$. (c) C. albicans $\mathrm{Dbf2}$ is required for proper nuclear segregation, actomyosin ring contraction, and cytokinesis ${ }^{38}$. A recent study involving the identification of Cdc14 interactome in C. albicans ${ }^{94}$ found only a subset of proteins $(0.2 \%)$ as physical or genetic interactors in S. cerevisiae, suggesting the divergence of Cdc14 functions in C. albicans. Hence, further investigations of MEN functioning in C. albicans are required to understand its divergence from S. cerevisiae and the mechanism by which Csa6 regulates mitotic exit in C. albicans and related species. Altogether, our results indicate that Csa6 has dual functions during cell cycle progression wherein it is first required during the G2/M phase for proper assembly of the mitotic spindle and then later during anaphase to exit the cells from mitosis. In addition, the constitutive localization of Csa6 to the SPBs strengthens the link between SPB-related functions and Csa6 in C. albicans (Fig. 8B, C).

The phylogenetic analysis of Csa6 revealed that it is only present in a group of fungal species, belonging to the CUG-Ser clade. Combined with its essential cell-cycle-related functions, it is intriguing to determine whether emergence of $\mathrm{Csa} 6$ is required to keep the pace of functional divergence in the regulatory mechanisms of cell cycle progression in these Candida species. While we demonstrated Csa6 of C. dubliniensis functionally complements Csa6 of C. albicans, whether Csa6 of distant species can also functionally complement CaCsa6 remains to be investigated. A recent study shows that around 50 essential genes, including Csa6, are only present in a group of Candida species (see Dataset 5 in ${ }^{95}$ ). Identification and functional characterization of these genes in the future will aid in developing clade-specific antifungal therapies ${ }^{95}$. In this study, we have analyzed only a part of the C. albicans ORFeome for their roles in genome maintenance. Further screening of the remaining overexpression ORFs will 
provide a complete network of the molecular pathways regulating genome stability in human fungal pathogens.

\section{Materials and Methods}

1. Strains, plasmids and primers. Information related to strains, plasmids and primers used in this study is available in the supplementary material.

2. Media and growth conditions. $C$. albicans strains were routinely grown at $30^{\circ} \mathrm{C}$ in $\mathrm{YPD}(1 \%$ yeast extract, $2 \%$ peptone, $2 \%$ dextrose $)$ medium supplemented with uridine $(0.1 \mu \mathrm{g} / \mathrm{ml})$ or complete medium (CM, 2\% dextrose, 1\% yeast nitrogen base and auxotrophic supplements) with or without uridine $(0.1 \mu \mathrm{g} / \mathrm{ml})$ and amino acids such as histidine, arginine, leucine $(0.1 \mu \mathrm{g} / \mathrm{ml})$. Solid media were prepared by adding $2 \%$ agar. For the selection of transformants, nourseothricin and hygromycin B (hyg B) were used at a final concentration of $100 \mu \mathrm{g} / \mathrm{ml}$ and $800 \mu \mathrm{g} / \mathrm{ml}$, respectively, in the YPDU medium.

Overexpression of genes from the tetracyline inducible promoter $\left(\mathrm{P}_{T E T}\right)$ was achieved by the addition of anhydrotetracycline (Atc, $3 \mu \mathrm{g} / \mathrm{ml}$ ) or doxycycline (Dox, $50 \mu \mathrm{g} / \mathrm{ml}$ ) in YPDU medium at $30^{\circ} \mathrm{C}{ }^{47}$ in the dark as Atc and Dox are light-sensitive. The $C S A 6^{P S D}$ strains were grown at $30^{\circ} \mathrm{C}$ either in permissive (YPDU) or nonpermissive (YPDU $+5 \mathrm{mM}$ methionine $(\mathrm{M})+5 \mathrm{mM}$ cysteine (C)) conditions of the MET3 promoter ${ }^{59,61}$. E. coli strains were cultured at $30^{\circ} \mathrm{C}$ or $37^{\circ} \mathrm{C}$ in Luria-Bertani (LB) medium or 2YT supplemented with ampicillin $(50 \mu \mathrm{g} / \mathrm{ml}$ or $100 \mu \mathrm{g} / \mathrm{ml})$, chloramphenicol $(34 \mu \mathrm{g} / \mathrm{ml})$, kanamycin $(50 \mu \mathrm{g} / \mathrm{ml})$ and tetracycline $(10 \mu \mathrm{g} / \mathrm{ml})$. Solid media were prepared by adding $2 \%$ agar. Chemically competent $E$. coli cells were prepared according to Chung et $a l^{96}$.

3. Flow cytometry analysis. Cultures of overexpression strains following $8 \mathrm{~h}$ of induction in YPDU+Atc and overnight recovery in the YPDU medium alone, were diluted in 1x phosphatebuffered saline (PBS) and analyzed ( $\sim 10^{6}$ cells) for the BFP/GFP marker by flow cytometry (FACSAria III, BD Biosciences) at a rate of 7000-10,000 events/s. We used 405- and 488-nm lasers to excite the BFP and GFP fluorophores and 450/40 and 530/30 filters to detect the BFP and GFP emission signals, respectively. 
4. Primary and secondary overexpression screening. To detect $\mathrm{CIN}$ at the BFP/GFP locus

upon $\mathrm{P}_{T E T}$ activation, overnight grown cultures of $C$. albicans overexpression strains were reinoculated in CM-His-Arg to ensure all cells contained BFP-HIS1 or GFP-ARG4. To measure the loss of BFP/GFP signals in 96-well plates, a $C D C 20^{O E}$ mutant was used as a positive control. The primary selection of the overexpression mutants with increased $\mathrm{BFP}^{+} \mathrm{GFP}^{-}$and $\mathrm{BFP}^{-} \mathrm{GFP}^{+}$ cells was done by determining the BFP/GFP loss frequency in EV. For this, we analyzed the flow cytometry density plots for 22 independent cultures of EV using the FlowJo software (FlowJo X 10.0.7r2). We observed a similar profile for all the cultures. We then defined gates for the $\mathrm{BFP}^{+} \mathrm{GFP}^{-}$and $\mathrm{BFP}^{-} \mathrm{GFP}^{+}$fractions of cell population in one of the EV samples and applied these gates to the rest of EV samples. The mean frequency of $\mathrm{BFP}^{+} \mathrm{GFP}^{-}$and $\mathrm{BFP}^{-} \mathrm{GFP}^{+}$cells in $\mathrm{EV}$ was calculated (Table S1). Similar gates were applied to all 1067 overexpression strains analyzed for $\mathrm{BFP} / \mathrm{GFP}$ markers and the frequency of $\mathrm{BFP}^{+} \mathrm{GFP}^{-}$and $\mathrm{BFP}^{-} \mathrm{GFP}^{+}$cells for each strain was determined (Dataset 1). The overexpression mutants, in which the BFP/GFP loss frequency was $\geq 2$-fold than EV, were selected for further analysis (Table S2).

For secondary screening, the overexpression plasmids present in each of the overexpression strains, identified from the primary screen (23 out of 1067), were used to retransform the CSA reporter strain (CEC5201). The overexpression strains (23) were analyzed by flow cytometry to revalidate the loss of BFP/GFP signals. Overexpression strains displaying $\geq 2$-fold higher frequency of $\mathrm{BFP}^{+} \mathrm{GFP}^{-} / \mathrm{BFP}^{-} \mathrm{GFP}^{+}$population than $\mathrm{EV}$ ( 6 out of 23 ) were monitored for any morphological transition by microscopy. As filamentous morphotype could distort the BFP/GFP loss analysis ${ }^{46}$, we characterized the overexpression mutants exhibiting increased CIN at the BFP/GFP locus and filamentous growth (3 out of 6 ) by monitoring cell cycle progression. For this, we transformed the overexpression plasmids in CaPJ159 and analyzed the overexpression strains (CSA4 ${ }^{M C M 7}, C S A 5^{B F A 1}$ and $C S A 6$ ) for DNA content, nuclear segregation and SPB separation. The 6 genes identified from the secondary screen were verified for the correct $C$. albicans ORF by Sanger sequencing using a common primer PJ90. During the secondary screening, we also cultured overexpression mutants in YPDU without Atc and observed no differences between EV and uninduced (-Atc) cultures in terms of morphology and the BFP/GFP loss frequency.

5. Cell sorting and marker analysis following a CIN event. Overnight grown cultures of EV and overexpression mutants $\left(C D C 20, C S A 1^{C L B 4}, C S A 2^{A S E 1}\right.$ and $\left.C S A 3^{K I P 2}\right)$ were reinoculated in YPDU+Atc for $8 \mathrm{~h}$ and allowed to recover overnight in YPDU-Atc. The cultures were analyzed 
for BFP/GFP loss by flow cytometry followed by fluorescence-activated cell sorting (FACS) using a cell sorter (FACSAria III, BD Biosciences) at a rate of 10,000 events/s. Approximately 1500 cells from the $\mathrm{BFP}^{-} \mathrm{GFP}^{+}$population were collected into $1.5-\mathrm{ml}$ tubes containing $400 \mu \mathrm{l}$ YPDU and immediately plated onto YPDU agar plates. Upon incubation at $30^{\circ} \mathrm{C}$ for 2 days, both small and large colonies appeared, as reported earlier (46). As most small colonies are expected to have undergone loss of the Ch4B haplotype (40), we analyzed auxotrophic/resistance markers of large colonies to characterize the molecular mechanisms underlying $\mathrm{CIN}$ in the overexpression mutants.

For marker analysis, we replica plated the large colonies along with the appropriate control strains on CM-Arg, CM-His and YPDU+hyg B $(800 \mu \mathrm{g} / \mathrm{ml})$ and incubated the plates at $30^{\circ} \mathrm{C}$ for 2 days. The colonies from CM-Arg plates were then analyzed for BFP, GFP and RFP markers by flow cytometry. For this, overnight grown cultures in YPDU were diluted in 1x PBS and 5000-10,000 cells were analyzed (FACSAria III, BD Biosciences). We used 405-, 488- and $561 \mathrm{~nm}$ lasers to excite the BFP, GFP and RFP fluorophores and 450/40, 530/30, 582/15 filters to detect the BFP, GFP and RFP emission signals, respectively.

6. Cell cycle analysis. Overnight grown cultures of $C$. albicans were reinoculated at an $\mathrm{OD}_{600}$ of 0.2 in different media (as described previously) and harvested at various time intervals postinoculation (as mentioned previously). The overnight grown culture itself was taken as a control sample $(0 \mathrm{~h})$ for all the experiments. Harvested samples were processed for propidium iodide (PI) staining as described before ${ }^{33}$. Stained cells were diluted to the desired cell density in $1 \mathrm{x}$ PBS and analyzed ( $\geq 30,000$ cells) by flow cytometry (FACSAria III, BD Biosciences) at a rate of 2501000 events/s. The output was analyzed using the FlowJo software (FlowJo X 10.0.7r2). We used 561-nm laser to excite PI and 610/20 filter to detect its emission signals.

7. Fluorescence microscopy. For nuclear division analysis in untagged strains, the C. albicans cells were grown overnight. The next day, the cells were transferred into different media (as mentioned previously) with a starting O.D. 600 of 0.2 , collected at various time intervals (as described previously) and fixed with formaldehyde (3.7\%). Cells were pelleted and washed thrice with $1 \mathrm{x}$ PBS, and Hoechst dye $(50 \mathrm{ng} / \mathrm{ml})$ was added to the cell suspension before imaging. Nuclear division in Cse4-and Tub4-tagged strains was analyzed as described above, except the cells were not fixed with formaldehyde. For Tem1 and mitotic spindle localization, overnight grown cultures were transferred to different media (as mentioned previously) with a starting 
O.D.600 of 0.2 and were grown for $6 \mathrm{~h}$ or $8 \mathrm{~h}$. Cells were then washed, resuspended in $1 \mathrm{x}$ PBS and imaged on a glass slide. Localization studies of each, CaCsa6, Tub4, Spc110 and CdCsa6 was carried out by washing the log phase grown cultures with 1x PBS (three times) followed by image acquisition.

The microscopy images were acquired using fluorescence microscope (Zeiss Axio Observer 7 equipped with Colibri 7 as the LED light source), 100x Plan Apochromat 1.4 NA objective, pco. edge 4.2 sCMOS. We used Zen 2.3 (blue edition) for image acquisition and controlling all hardware components. Filter set $92 \mathrm{HE}$ with excitation 455-483 and 583-600 nm for GFP and mCherry, respectively, and corresponding emission was captured at 501-547 and 617-758 nm. Z sections were obtained at an interval of $300 \mathrm{~nm}$. All the images were displayed after the maximum intensity projection using ImageJ. Image processing was done using ImageJ. We used the cell counter plugin of ImageJ to count various cell morphologies in different mutant strains. Images acquired in the mCherry channel were processed using the subtract background plugin of ImageJ for better visualization.

8. Protein preparation and western blotting. Approximately 3 O.D.600 equivalent cells were taken, washed with water once and resuspended in 12.5\% TCA (trichloroacetic acid) and incubated at $-20^{\circ} \mathrm{C}$ overnight for precipitation. The cells were pelleted down and washed twice with ice-cold $80 \%$ acetone. The pellet was then allowed to air dry and finally resuspended in lysis buffer $\left(0.1 \mathrm{~N} \mathrm{NaOH}\right.$ and $1 \%$ SDS and 5xprotein loading dye). Samples were boiled at $95^{\circ} \mathrm{C}$ for 5 10 min and electrophoresed on a 10\% SDS polyacrylamide gel. Gels were transferred to a nitrocellulose membrane by semi-dry method for $30 \mathrm{~min}$ at $25 \mathrm{~V}$ and blocked for an hour in $5 \%$ non-fat milk in 1x PBS. Membranes were incubated with a 1:5000 dilution of rabbit anti-Protein A or mouse anti-PSTAIRE in 2.5\% non-fat milk in 1x PBS. Membranes were washed three times in 1x PBS-Tween (0.05\%) and then exposed to a 1:10,000 dilution of either anti-mouse- or antirabbit-IgG horseradish peroxidase antibody in $2.5 \%$ non-fat milk in 1x PBS. Membranes were washed three times in 1x PBS-Tween $(0.05 \%)$ and developed using chemiluminescence method.

9. Statistical analysis. Statistical significance of differences was calculated as mentioned in the figure legends with unpaired one-tailed $t$-test, paired one-tailed $t$-test, paired two-tailed $t$-test or one-way ANOVA with Bonferroni posttest. $P$-values $\geq 0.05$ were considered as nonsignificant (n.s.). $P$-values of the corresponding figures are mentioned, if significant. All analyses were conducted using GraphPad Prism version Windows v5.00. 


\section{References}

1. Aguilera A, Gomez-Gonzalez B. Genome instability: a mechanistic view of its causes and consequences. Nat Rev Genet 9, 204-217 (2008).

2. Potapova TA, Zhu J, Li R. Aneuploidy and chromosomal instability: a vicious cycle driving cellular evolution and cancer genome chaos. Cancer Metastasis Rev 32, 377-389 (2013).

3. Yurov YB, Vorsanova SG, Iourov IY. Chromosome Instability in the Neurodegenerating Brain. Front Genet 10, 892 (2019).

4. Taylor AMR, et al. Chromosome instability syndromes. Nat Rev Dis Primers 5, 64 (2019).

5. Petr MA, Tulika T, Carmona-Marin LM, Scheibye-Knudsen M. Protecting the Aging Genome. Trends Cell Biol 30, 117-132 (2020).

6. Negrini S, Gorgoulis VG, Halazonetis TD. Genomic instability--an evolving hallmark of cancer. Nat Rev Mol Cell Biol 11, 220-228 (2010).

7. Guin K, et al. Spatial inter-centromeric interactions facilitated the emergence of evolutionary new centromeres. Elife 9, (2020).

8. Sankaranarayanan SR, et al. Loss of centromere function drives karyotype evolution in closely related Malassezia species. Elife 9, (2020).

9. Aguilera A, Garcia-Muse T. Causes of genome instability. Annu Rev Genet 47, 1-32 (2013).

10. Levine MS, Holland AJ. The impact of mitotic errors on cell proliferation and tumorigenesis. Genes Dev 32, 620-638 (2018).

11. Yuen KW, Warren CD, Chen O, Kwok T, Hieter P, Spencer FA. Systematic genome instability screens in yeast and their potential relevance to cancer. Proc Natl Acad Sci US A 104, 3925-3930 (2007).

12. Stirling PC, et al. The complete spectrum of yeast chromosome instability genes identifies candidate CIN cancer genes and functional roles for ASTRA complex components. PLoS Genet 7, e1002057 (2011).

13. Stevenson LF, Kennedy BK, Harlow E. A large-scale overexpression screen in Saccharomyces cerevisiae identifies previously uncharacterized cell cycle genes. Proc Natl Acad Sci U S A 98, 3946-3951 (2001).

14. Duffy S, et al. Overexpression screens identify conserved dosage chromosome instability genes in yeast and human cancer. Proc Natl Acad Sci U S A 113, 9967-9976 (2016). 
15. Espinet C, de la Torre MA, Aldea M, Herrero E. An efficient method to isolate yeast genes causing overexpression-mediated growth arrest. Yeast 11, 25-32 (1995).

16. Akada R, Yamamoto J, Yamashita I. Screening and identification of yeast sequences that cause growth inhibition when overexpressed. Mol Gen Genet 254, 267-274 (1997).

17. Legrand M, Jaitly P, Feri A, d'Enfert C, Sanyal K. Candida albicans: An Emerging Yeast Model to Study Eukaryotic Genome Plasticity. Trends Genet 35, 292-307 (2019).

18. Brown GD, Denning DW, Gow NA, Levitz SM, Netea MG, White TC. Hidden killers: human fungal infections. Sci Transl Med 4, 165rv113 (2012).

19. Friedman DZP, Schwartz IS. Emerging Fungal Infections: New Patients, New Patterns, and New Pathogens. J Fungi (Basel) 5, (2019).

20. Selmecki A, Forche A, Berman J. Genomic plasticity of the human fungal pathogen Candida albicans. Eukaryot Cell 9, 991-1008 (2010).

21. Forche A, Alby K, Schaefer D, Johnson AD, Berman J, Bennett RJ. The parasexual cycle in Candida albicans provides an alternative pathway to meiosis for the formation of recombinant strains. PLoS Biol 6, e110 (2008).

22. Feri A, et al. Analysis of Repair Mechanisms following an Induced Double-Strand Break Uncovers Recessive Deleterious Alleles in the Candida albicans Diploid Genome. mBio 7, (2016).

23. Selmecki A, Gerami-Nejad M, Paulson C, Forche A, Berman J. An isochromosome confers drug resistance in vivo by amplification of two genes, ERG11 and TAC1. Mol Microbiol 68, 624-641 (2008).

24. Dunkel N, Blass J, Rogers PD, Morschhauser J. Mutations in the multi-drug resistance regulator MRR1, followed by loss of heterozygosity, are the main cause of MDR1 overexpression in fluconazole-resistant Candida albicans strains. Mol Microbiol 69, 827840 (2008).

25. Selmecki AM, Dulmage K, Cowen LE, Anderson JB, Berman J. Acquisition of aneuploidy provides increased fitness during the evolution of antifungal drug resistance. PLoS Genet 5, e1000705 (2009).

26. Ford CB, et al. The evolution of drug resistance in clinical isolates of Candida albicans. Elife 4, e00662 (2015).

27. Selmecki A, Forche A, Berman J. Aneuploidy and isochromosome formation in drugresistant Candida albicans. Science 313, 367-370 (2006).

28. Coste A, et al. A mutation in Tac1p, a transcription factor regulating CDR1 and CDR2, is coupled with loss of heterozygosity at chromosome 5 to mediate antifungal resistance in Candida albicans. Genetics 172, 2139-2156 (2006). 
29. Forche A, et al. Rapid Phenotypic and Genotypic Diversification After Exposure to the Oral Host Niche in Candida albicans. Genetics 209, 725-741 (2018).

30. Tso GHW, et al. Experimental evolution of a fungal pathogen into a gut symbiont. Science 362, 589-595 (2018).

31. Forche A, Magee PT, Selmecki A, Berman J, May G. Evolution in Candida albicans populations during a single passage through a mouse host. Genetics 182, 799-811 (2009).

32. Bennett RJ, Forche A, Berman J. Rapid mechanisms for generating genome diversity: whole ploidy shifts, aneuploidy, and loss of heterozygosity. Cold Spring Harb Perspect Med 4, (2014).

33. Sanyal K, Carbon J. The CENP-A homolog CaCse4p in the pathogenic yeast Candida albicans is a centromere protein essential for chromosome transmission. Proc Natl Acad Sci U S A 99, 12969-12974 (2002).

34. Sanyal K, Baum M, Carbon J. Centromeric DNA sequences in the pathogenic yeast Candida albicans are all different and unique. Proc Natl Acad Sci U S A 101, 1137411379 (2004).

35. Guin K, Sreekumar L, Sanyal K. Implications of the Evolutionary Trajectory of Centromeres in the Fungal Kingdom. Annu Rev Microbiol 74, 835-853 (2020).

36. Musacchio A, Desai A. A Molecular View of Kinetochore Assembly and Function. Biology (Basel) 6, (2017).

37. Varshney N, Sanyal K. Nuclear migration in budding yeasts: position before division. Curr Genet 65, 1341-1346 (2019).

38. Gonzalez-Novo A, et al. Dbf2 is essential for cytokinesis and correct mitotic spindle formation in Candida albicans. Mol Microbiol 72, 1364-1378 (2009).

39. Milne SW, et al. Role of Candida albicans Tem1 in mitotic exit and cytokinesis. Fungal Genet Biol 69, 84-95 (2014).

40. Bates S. Candida albicans Cdc15 is essential for mitotic exit and cytokinesis. Sci Rep 8, 8899 (2018).

41. Thakur J, Sanyal K. The essentiality of the fungus-specific Dam1 complex is correlated with a one-kinetochore-one-microtubule interaction present throughout the cell cycle, independent of the nature of a centromere. Eukaryot Cell 10, 1295-1305 (2011).

42. Roy B, Burrack LS, Lone MA, Berman J, Sanyal K. CaMtw1, a member of the evolutionarily conserved Mis 12 kinetochore protein family, is required for efficient inner kinetochore assembly in the pathogenic yeast Candida albicans. Mol Microbiol 80, 14-32 (2011). 
43. Legrand M, Chan CL, Jauert PA, Kirkpatrick DT. Role of DNA mismatch repair and double-strand break repair in genome stability and antifungal drug resistance in Candida albicans. Eukaryot Cell 6, 2194-2205 (2007).

44. Legrand M, Chan CL, Jauert PA, Kirkpatrick DT. The contribution of the S-phase checkpoint genes MEC1 and SGS1 to genome stability maintenance in Candida albicans. Fungal Genet Biol 48, 823-830 (2011).

45. Loll-Krippleber R, et al. A study of the DNA damage checkpoint in Candida albicans: uncoupling of the functions of Rad53 in DNA repair, cell cycle regulation and genotoxic stress-induced polarized growth. Mol Microbiol 91, 452-471 (2014).

46. Loll-Krippleber R, et al. A FACS-optimized screen identifies regulators of genome stability in Candida albicans. Eukaryot Cell 14, 311-322 (2015).

47. Chauvel M, et al. A versatile overexpression strategy in the pathogenic yeast Candida albicans: identification of regulators of morphogenesis and fitness. PLoS One 7, e45912 (2012).

48. Bachewich C, Nantel A, Whiteway M. Cell cycle arrest during S or M phase generates polarized growth via distinct signals in Candida albicans. Mol Microbiol 57, 942-959 (2005).

49. Bensen ES, Clemente-Blanco A, Finley KR, Correa-Bordes J, Berman J. The mitotic cyclins Clb2p and Clb4p affect morphogenesis in Candida albicans. Mol Biol Cell 16, 3387-3400 (2005).

50. Thakur J, Sanyal K. A coordinated interdependent protein circuitry stabilizes the kinetochore ensemble to protect CENP-A in the human pathogenic yeast Candida albicans. PLoS Genet 8, e1002661 (2012).

51. Kitamura E, Tanaka K, Kitamura Y, Tanaka TU. Kinetochore microtubule interaction during S phase in Saccharomyces cerevisiae. Genes Dev 21, 3319-3330 (2007).

52. Jin QW, Fuchs J, Loidl J. Centromere clustering is a major determinant of yeast interphase nuclear organization. J Cell Sci 113 ( Pt 11), 1903-1912 (2000).

53. Lin TC, Neuner A, Schiebel E. Targeting of gamma-tubulin complexes to microtubule organizing centers: conservation and divergence. Trends Cell Biol 25, 296-307 (2015).

54. Lin TC, et al. MOZART1 and gamma-tubulin complex receptors are both required to turn gamma-TuSC into an active microtubule nucleation template. J Cell Biol 215, 823-840 (2016).

55. Musacchio A, Salmon ED. The spindle-assembly checkpoint in space and time. Nat Rev Mol Cell Biol 8, 379-393 (2007).

56. Kops G, Snel B, Tromer EC. Evolutionary Dynamics of the Spindle Assembly Checkpoint in Eukaryotes. Curr Biol 30, R589-R602 (2020). 
57. Caydasi AK, Pereira G. SPOC alert--when chromosomes get the wrong direction. Exp Cell Res 318, 1421-1427 (2012).

58. Scarfone I, Piatti S. Coupling spindle position with mitotic exit in budding yeast: The multifaceted role of the small GTPase Tem1. Small GTPases 6, 196-201 (2015).

59. Care RS, Trevethick J, Binley KM, Sudbery PE. The MET3 promoter: a new tool for Candida albicans molecular genetics. Mol Microbiol 34, 792-798 (1999).

60. Shivaraju M, Unruh JR, Slaughter BD, Mattingly M, Berman J, Gerton JL. Cell-cyclecoupled structural oscillation of centromeric nucleosomes in yeast. Cell 150, 304-316 (2012).

61. Sreekumar L, et al. Orc4 spatiotemporally stabilizes centromeric chromatin. Genome Res 31, 607-621 (2021).

62. Liu HY, Toyn JH, Chiang YC, Draper MP, Johnston LH, Denis CL. DBF2, a cell cycleregulated protein kinase, is physically and functionally associated with the CCR4 transcriptional regulatory complex. EMBO J 16, 5289-5298 (1997).

63. Surana U, Amon A, Dowzer C, McGrew J, Byers B, Nasmyth K. Destruction of the $\mathrm{CDC} 28 / \mathrm{CLB}$ mitotic kinase is not required for the metaphase to anaphase transition in budding yeast. EMBO J 12, 1969-1978 (1993).

64. Hotz M, Barral Y. The Mitotic Exit Network: new turns on old pathways. Trends Cell Biol 24, 145-152 (2014).

65. Atir-Lande A, Gildor T, Kornitzer D. Role for the SCFCDC4 ubiquitin ligase in Candida albicans morphogenesis. Mol Biol Cell 16, 2772-2785 (2005).

66. Shirayama M, Matsui Y, Toh EA. The yeast TEM1 gene, which encodes a GTP-binding protein, is involved in termination of M phase. Mol Cell Biol 14, 7476-7482 (1994).

67. Valerio-Santiago M, Monje-Casas F. Tem1 localization to the spindle pole bodies is essential for mitotic exit and impairs spindle checkpoint function. J Cell Biol 192, 599-614 (2011).

68. Lee SE, Frenz LM, Wells NJ, Johnson AL, Johnston LH. Order of function of the budding-yeast mitotic exit-network proteins Tem1, Cdc15, Mob1, Dbf2, and Cdc5. Curr Biol 11, 784-788 (2001).

69. Jackson AP, et al. Comparative genomics of the fungal pathogens Candida dubliniensis and Candida albicans. Genome Res 19, 2231-2244 (2009).

70. Padmanabhan S, Thakur J, Siddharthan R, Sanyal K. Rapid evolution of Cse4p-rich centromeric DNA sequences in closely related pathogenic yeasts, Candida albicans and Candida dubliniensis. Proc Natl Acad Sci U S A 105, 19797-19802 (2008).

71. Chatterjee G, et al. Repeat-Associated Fission Yeast-Like Regional Centromeres in the Ascomycetous Budding Yeast Candida tropicalis. PLoS Genet 12, e1005839 (2016). 
72. Bijlani S, Thevandavakkam MA, Tsai HJ, Berman J. Autonomously Replicating Linear Plasmids That Facilitate the Analysis of Replication Origin Function in Candida albicans. mSphere 4, (2019).

73. Pellman D, Bagget M, Tu YH, Fink GR, Tu H. Two microtubule-associated proteins required for anaphase spindle movement in Saccharomyces cerevisiae. J Cell Biol 130, 1373-1385 (1995).

74. Schuyler SC, Liu JY, Pellman D. The molecular function of Ase1p: evidence for a MAPdependent midzone-specific spindle matrix. Microtubule-associated proteins. $J$ Cell Biol 160, 517-528 (2003).

75. Liu H, Liang F, Jin F, Wang Y. The coordination of centromere replication, spindle formation, and kinetochore-microtubule interaction in budding yeast. PLoS Genet $\mathbf{4}$, e1000262 (2008).

76. Wang Y, Hu F, Elledge SJ. The Bfa1/Bub2 GAP complex comprises a universal checkpoint required to prevent mitotic exit. Curr Biol 10, 1379-1382 (2000).

77. Ro HS, Song S, Lee KS. Bfa1 can regulate Tem1 function independently of Bub2 in the mitotic exit network of Saccharomyces cerevisiae. Proc Natl Acad Sci U S A 99, 54365441 (2002).

78. Ofir A, Kornitzer D. Candida albicans cyclin Clb4 carries S-phase cyclin activity. Eukaryot Cell 9, 1311-1319 (2010).

79. Schwob E, Nasmyth K. CLB5 and CLB6, a new pair of B cyclins involved in DNA replication in Saccharomyces cerevisiae. Genes Dev 7, 1160-1175 (1993).

80. Hibbel A, et al. Kinesin Kip2 enhances microtubule growth in vitro through lengthdependent feedback on polymerization and catastrophe. Elife 4, (2015).

81. Augustine B, Chin CF, Yeong FM. Role of Kip2 during early mitosis - impact on spindle pole body separation and chromosome capture. J Cell Sci 131, (2018).

82. Riera A, Barbon M, Noguchi Y, Reuter LM, Schneider S, Speck C. From structure to mechanism-understanding initiation of DNA replication. Genes Dev 31, 1073-1088 (2017).

83. Labib K, Tercero JA, Diffley JF. Uninterrupted MCM2-7 function required for DNA replication fork progression. Science 288, 1643-1647 (2000).

84. Ren B, et al. MCM7 amplification and overexpression are associated with prostate cancer progression. Oncogene 25, 1090-1098 (2006).

85. Toyokawa G, et al. Minichromosome Maintenance Protein 7 is a potential therapeutic target in human cancer and a novel prognostic marker of non-small cell lung cancer. Mol Cancer 10, 65 (2011). 
86. Qiu YT, Wang WJ, Zhang B, Mei LL, Shi ZZ. MCM7 amplification and overexpression promote cell proliferation, colony formation and migration in esophageal squamous cell carcinoma by activating the AKT1/mTOR signaling pathway. Oncol Rep 37, 3590-3596 (2017).

87. Weinert TA, Hartwell LH. The RAD9 gene controls the cell cycle response to DNA damage in Saccharomyces cerevisiae. Science 241, 317-322 (1988).

88. Waterman DP, Haber JE, Smolka MB. Checkpoint Responses to DNA Double-Strand Breaks. Annu Rev Biochem 89, 103-133 (2020).

89. Sridhar S, Hori T, Nakagawa R, Fukagawa T, Sanyal K. Bridgin connects the outer kinetochore to centromeric chromatin. Nat Commun 12, 146 (2021).

90. Zhang Y, et al. USP44 regulates centrosome positioning to prevent aneuploidy and suppress tumorigenesis. J Clin Invest 122, $4362-4374$ (2012).

91. Silkworth WT, Nardi IK, Paul R, Mogilner A, Cimini D. Timing of centrosome separation is important for accurate chromosome segregation. Mol Biol Cell 23, 401-411 (2012).

92. Nam HJ, Naylor RM, van Deursen JM. Centrosome dynamics as a source of chromosomal instability. Trends Cell Biol 25, 65-73 (2015).

93. Clemente-Blanco A, et al. The Cdc14p phosphatase affects late cell-cycle events and morphogenesis in Candida albicans. J Cell Sci 119, 1130-1143 (2006).

94. Kaneva IN, Sudbery IM, Dickman MJ, Sudbery PE. Proteins that physically interact with the phosphatase Cdc14 in Candida albicans have diverse roles in the cell cycle. Sci Rep 9, 6258 (2019).

95. Segal ES, et al. Gene Essentiality Analyzed by In Vivo Transposon Mutagenesis and Machine Learning in a Stable Haploid Isolate of Candida albicans. mBio 9, (2018).

96. Chung CT, Niemela SL, Miller RH. One-step preparation of competent Escherichia coli: transformation and storage of bacterial cells in the same solution. Proc Natl Acad Sci US A 86, 2172-2175 (1989).

97. Potter SC, Luciani A, Eddy SR, Park Y, Lopez R, Finn RD. HMMER web server: 2018 update. Nucleic Acids Res 46, W200-W204 (2018).

98. Luca FC, Mody M, Kurischko C, Roof DM, Giddings TH, Winey M. Saccharomyces cerevisiae Mob1p is required for cytokinesis and mitotic exit. Mol Cell Biol 21, 69726983 (2001).

99. Tamborrini D, Juanes MA, Ibanes S, Rancati G, Piatti S. Recruitment of the mitotic exit network to yeast centrosomes couples septin displacement to actomyosin constriction. Nat Commun 9, 4308 (2018).

100. Sopko R, et al. Mapping pathways and phenotypes by systematic gene overexpression. Mol Cell 21, 319-330 (2006). 
101. Li R. Bifurcation of the mitotic checkpoint pathway in budding yeast. Proc Natl Acad Sci U S A 96, 4989-4994 (1999).

\section{Acknowledgments}

We thank members of the Sanyal and d'Enfert laboratories for their valuable suggestions and constructive criticism. We thank the Munro group at University of Aberdeen and Mazel group at Institut Pasteur for their contribution to the establishment of overexpression plasmids that were used in this study, a work that will be reported elsewhere. We thank Dr. Arshad Desai for critical reading of the manuscript. We thank N. Varshney for constructing the plasmid pCse4-TAP-Leu. We thank L. Sreekumar for constructing pTub4-GFP-His cassette. Special thanks to K. Guin for sharing the raw files to generate the phylogenetic tree. We thank V. Sood and A. Das for generating the plasmid pCdCsa6-GFP-ARS2. We thank A.S. Amrutha for generating the strains CaPJ300 and CaPJ301. We acknowledge N. Nala at the flow cytometry facility, JNCASR, for assisting flow cytometry and cell sorting experiments. The establishment of overexpression plasmids was supported by the Wellcome Trust $[088858 / \mathrm{Z} / 09 / \mathrm{Z}$ to CD]. This work was supported by a grant from the Indo French Centre for the promotion of Advanced Research (CEFIPRA, Project no. 5703-2). CEFIPRA also aided in the travel of PJ, KS and CD between the Sanyal and d'Enfert laboratories. PJ acknowledges intramural funding from JNCASR. AD and TP were supported by the CEFIPRA grant. K.S. acknowledges the financial support of JC Bose National Fellowship (Science and Engineering Research Board, Govt. of India, JCB/2020/000021) and intramural funding from JNCASR.

\section{Funding}

Indo French Centre for the promotion of Advanced Research (CEFIPRA, Project no. 5703-2). Jawaharlal Nehru Centre for Advanced Scientific Research.

JC Bose National Fellowship (Science and Engineering Research Board, Govt. of India, $\mathrm{JCB} / 2020 / 000021)$.

The Wellcome Trust (088858/Z/09/Z).

Institut Pasteur.

Institut national de la recherche pour l'agriculture, l'alimentation et l'environnement (INRAE). 
1021 Author contributions:

1022 Conceptualization: KS, CD, PJ, ML

1023 Methodology: ML, PJ, AD, TP, MC

1024 Investigation: PJ, AD, TP, ML

1025 Supervision: KS, CD, ML

1026 Writing — original draft: PJ, KS

1027 Writing — review \& editing: PJ, KS, CD, ML

1028

1029 Competing interests: The authors declare no competing interests.

1030

Data and materials availability: All data are available in the main text or supplementary materials. 
A

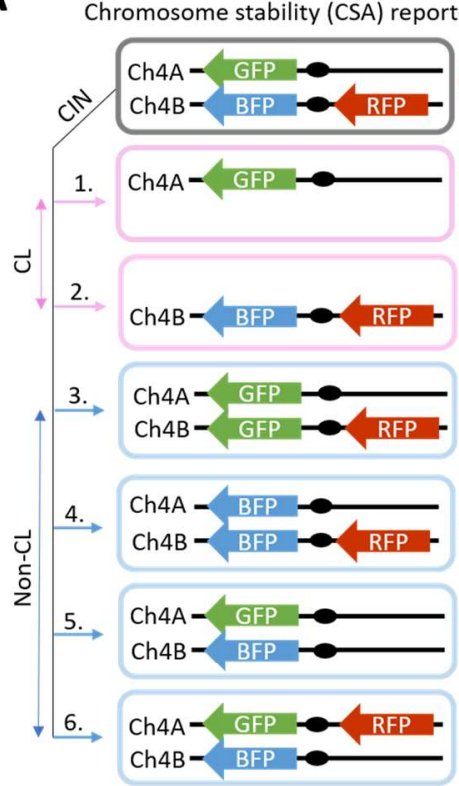

B

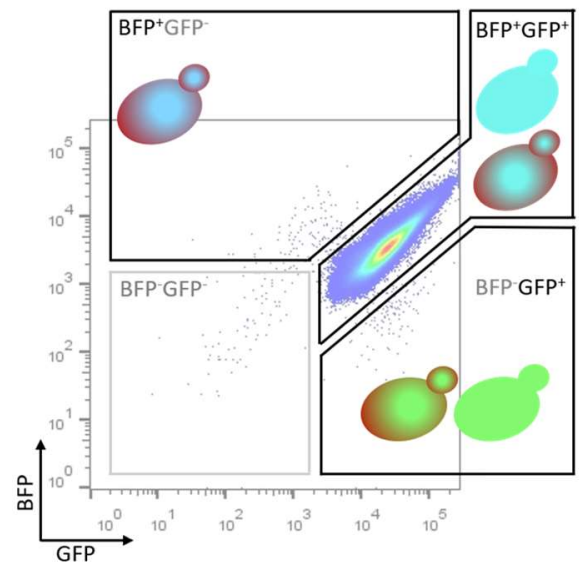

C

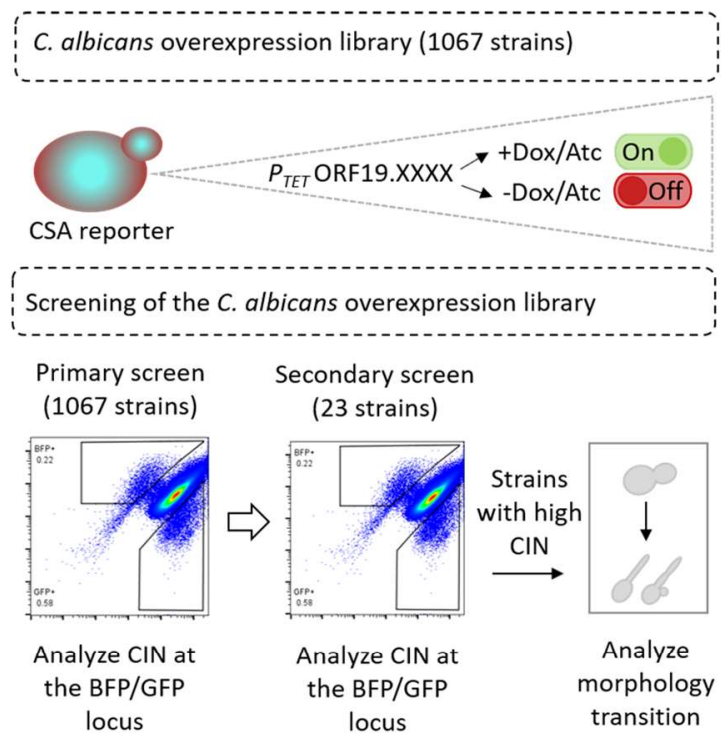

D

\begin{tabular}{|c|c|c|c|c|}
\hline CSA gene & $\begin{array}{l}\text { S. cerevisiae } \\
\text { homolog }\end{array}$ & $\begin{array}{l}\text { C. albicans } \\
\text { ORF no. }\end{array}$ & $\begin{array}{l}\text { ORF status in } \\
\text { C. albicans }\end{array}$ & Description \\
\hline CSA1 & CLB4 & 19.7186 & Verified & $\begin{array}{l}\text { B-type mitotic } \\
\text { cyclin }\end{array}$ \\
\hline CSA2 & ASE1 & 19.7377 & Predicted & $\begin{array}{l}\text { Microtubule- } \\
\text { associated protein }\end{array}$ \\
\hline CSA3 & KIP2 & 19.1747 & Predicted & $\begin{array}{l}\text { Kinesin-related } \\
\text { motor protein }\end{array}$ \\
\hline CSA4 & MCM7 & 19.202 & Predicted & $\begin{array}{l}\text { Component of } \\
\text { Mcm2-7 complex }\end{array}$ \\
\hline CSA5 & BFA1 & 19.6080 & Predicted & $\begin{array}{c}\text { GTPase activating } \\
\text { protein }\end{array}$ \\
\hline CSA & Absent & 19.1447 & Predicted & - \\
\hline
\end{tabular}

Fig. 1. A medium-throughput protein overexpression screen identifies a set of $C S A$ genes in GFP locus, because of either chromosome loss (CL) or non-CL events such as break-induced replication, gene conversion, chromosome truncation or mitotic crossing over, will lead to the expression of either GFP or BFP expressing genes. CIN due to CL can be specifically identified by the concomitant loss of BFP and RFP, as shown in 1.5 and 6, cells undergoing non-CL events at the RFP locus will continue to express BFP and GFP. (B) Flow cytometric analysis of the BFP/GFP density profile of empty vector (EV) (CaPJ150) containing BFP, GFP and RFP genes. Majority of the cells are positive for both BFP and GFP $\left(\mathrm{BFP}^{+} \mathrm{GFP}^{+}\right)$. A minor fraction of the population had lost either one of the markers $\left(\mathrm{BFP}^{+} \mathrm{GFP}^{-}\right.$or $\left.\mathrm{BFP}^{-} \mathrm{GFP}^{+}\right)$or both the markers $\left(\mathrm{BFP}^{-}\right.$ 
$1068 \mathrm{GFP}^{-}$), indicating spontaneous instability of this locus ${ }^{46}$. Approximately 1 million events are 1069 displayed. (C) Pictorial representation of the screening strategy employed for identifying CSA 1070 genes in C. albicans. Briefly, a library of C. albicans overexpression strains (1067), each carrying 1071 a unique ORF under the tetracycline-inducible promoter, $\mathrm{P}_{T E T}$, was generated using the CSA 1072 reporter (CEC5201) as the parent strain. The library was then analyzed by primary and secondary 1073 screening methods to identify CSA genes. In the primary screen, CIN frequency at the BFP/GFP

1074 locus in the individual 1067 overexpression strains was determined using flow cytometry.

1075 Overexpression strains exhibiting increased CIN (23 out of 1067) were taken forward for 1076 secondary screening. The secondary screen involved revalidation of the primary hits for increased

$1077 \mathrm{CIN}$ at the BFP/GFP locus by flow cytometry. Strains which reproduced the increased CIN 1078 phenotype were further examined for yeast to filamentous transition by microscopy. (D) A brief 1079 overview of the CSA genes identified from the overexpression screen (6 out of 1067). Functional 1080 annotation of genes is based on the information available either in Candida Genome Database 1081 (www.candidagenome.org) or in Saccharomyces Genome Database (www.yeastgenome.org) on 1082 August 1, 2021. 

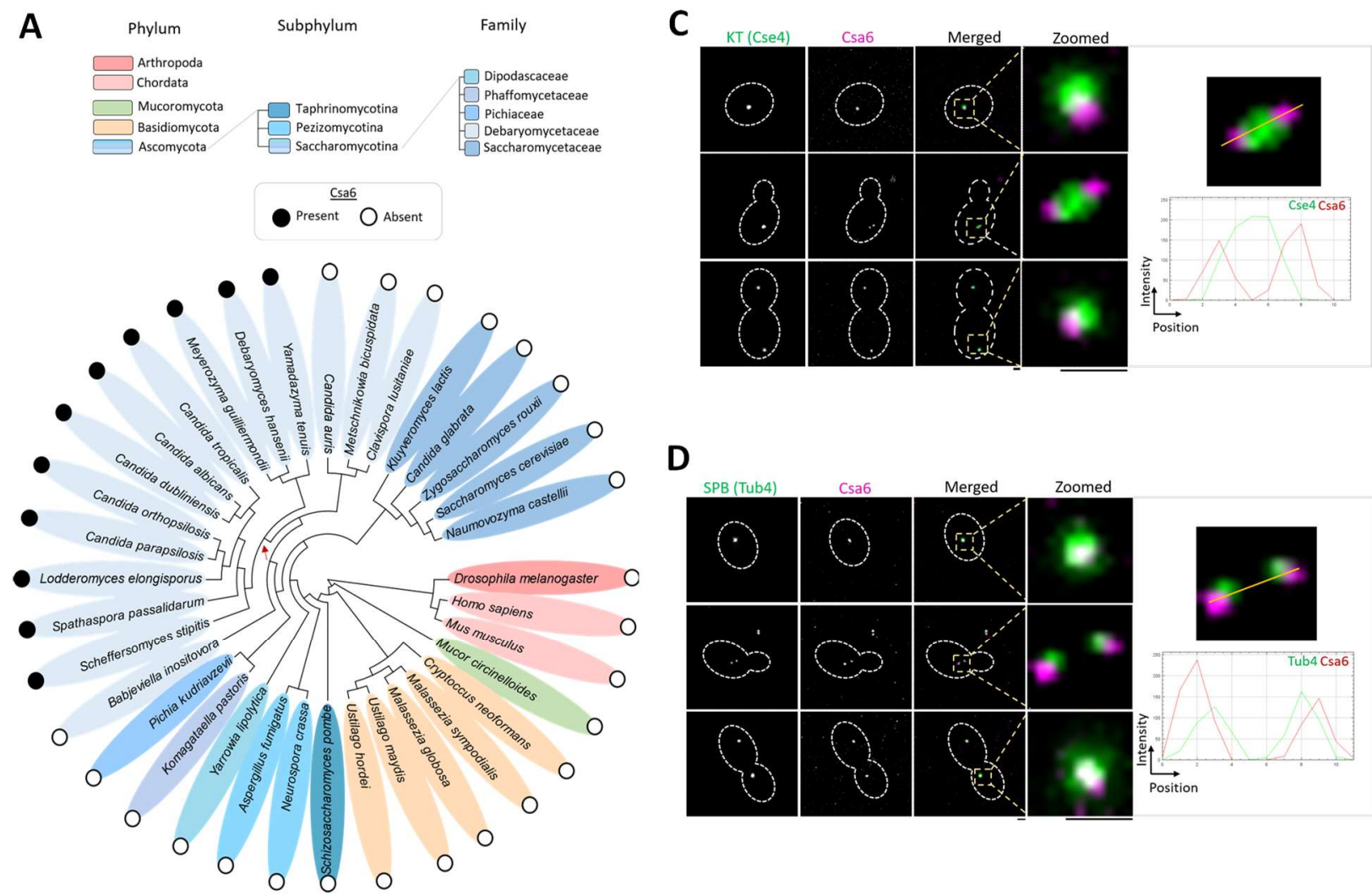

B

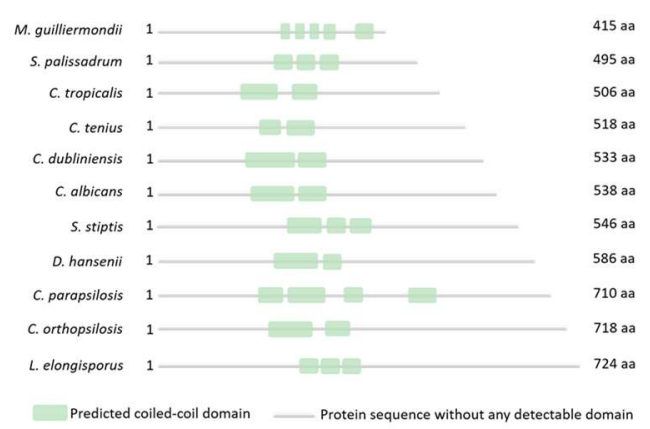

\section{E}

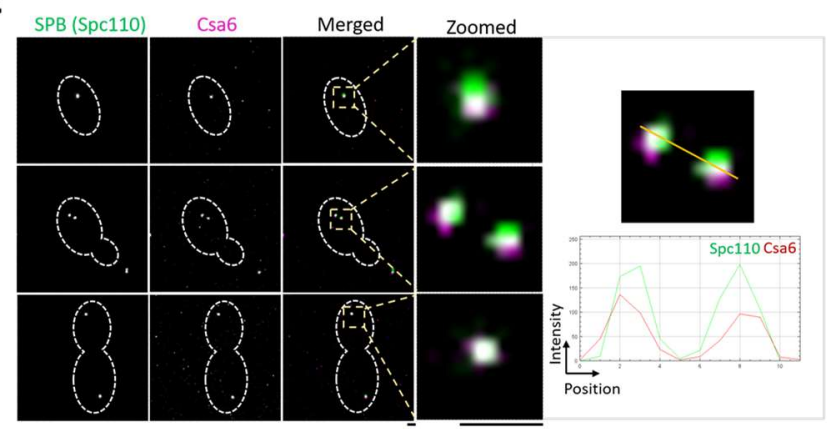

Fig. 2. Csa6 has a selective existence across fungal phylogeny and is constitutively localized

to the SPBs in $C$. albicans. (A) Phylogenetic tree showing the conservation of Csa6 across the mentioned species. The presence (filled circles) or absence (empty circles) of Csa6 in every species is marked. Each taxonomic rank is color-coded. The species mentioned under the family Debaryomycetaceae belong to the CUG-Ser clade in which the CUG codon is often translated as serine instead of leucine. The red arrow points to the CUG-Ser clade lineage that acquired Csa6.

1112 Searches for Csa6 homologs $\left(E\right.$ value $\left.\leq 10^{-2}\right)$ were carried out either in the Candida Genome Database (www.candidagenome.org) or NCBI nonredundant protein database. (B) Schematic illustrating the protein domain architecture alignment of Csa6 in the indicated fungal species. 
1115 Length of the protein is mentioned as amino acids (aa). Approximate positions of the predicted

1116 coiled-coil domain, identified using HMMER ${ }^{97}$ phmmer searches, is shown. (C-E) Left,

1117 micrographs comparing the sub-cellular localization of Csa6 with KT (Cse4) and SPB (Tub4 and

1118 Spc110) at various cell cycle stages. Top, Csa6-mCherry and Cse4-GFP (CaPJ119); middle, Csa6-

1119 mCherry and Tub4-GFP (CaPJ120), and bottom, Csa6m-Cherry and Spc110-GFP (CaPJ121).

1120 Scale bar, $1 \mu \mathrm{m}$. Right, histogram plots showing the fluorescence intensity profile of Csa6-

1121 mCherry with Cse4-GFP (top), Tub4-GFP (middle) and Spc110-GFP (bottom) across the 1122 indicated lines. 
Figure 3

A

$$
P_{T E T} C S A 6\left\{\begin{array}{l}
+ \text { Dox/Atc On } \\
\text {-Dox/Atc OOff }
\end{array}\right.
$$

B

Western blot: Csa6-TAP

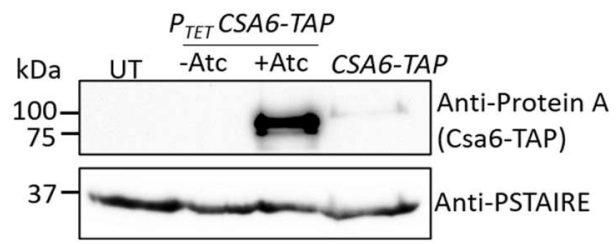

C

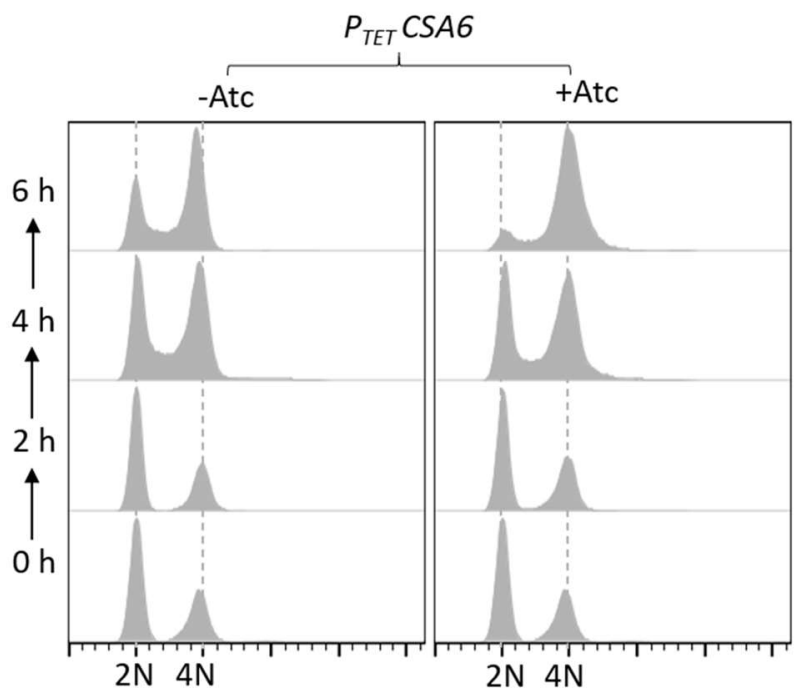

D
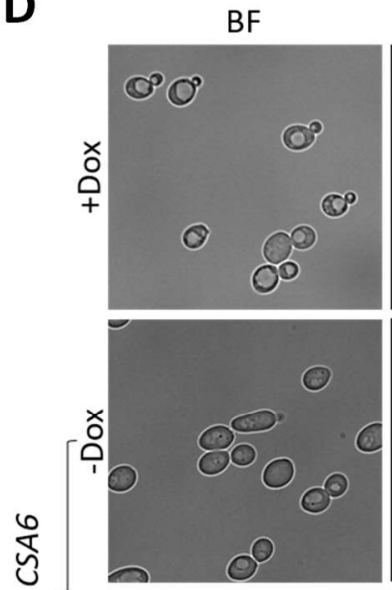

这

1146

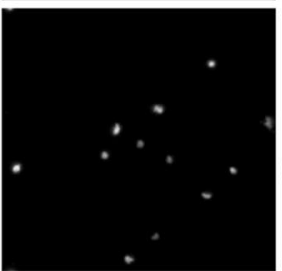

Merged

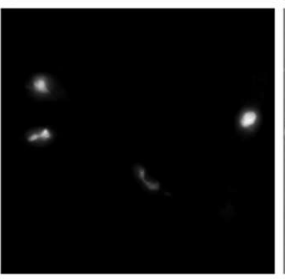

E
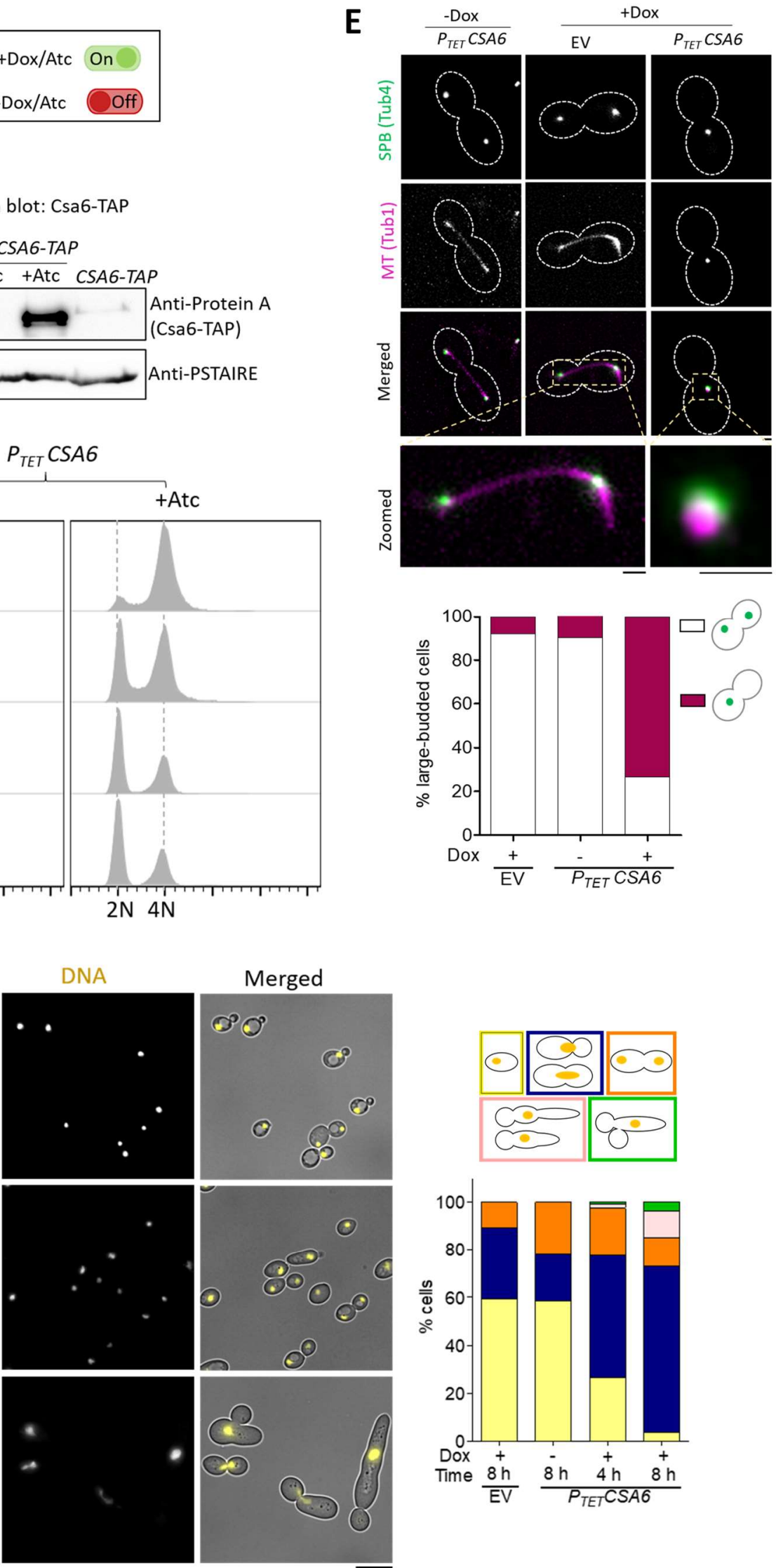
Fig. 3. Overexpression of Csa6 alters the morphology of the mitotic spindle and leads to

G2/M arrest in $C$. albicans. (A) Atc/Dox-dependent functioning of the $\mathrm{P}_{T E T}$ promoter system for conditional overexpression of CSA6. (B) Western blot analysis using anti-Protein A antibodies confirmed overexpression of CSA6-TAP from the $\mathrm{P}_{\text {TET }}$ promoter (CaPJ181), after $8 \mathrm{~h}$ induction in presence of Atc $(3 \mu \mathrm{g} / \mathrm{ml})$, in comparison to the uninduced culture (-Atc) or CSA6-TAP expression from its native promoter (CaPJ180); $N=2$. PSTAIRE was used as a loading control. UT, untagged control (SN148). (C) DNA content of $C S A 6^{O E}$ strain $(\mathrm{CaPJ} 176)$ in presence or absence of Atc $(3 \mu \mathrm{g} / \mathrm{ml})$ at the indicated time intervals; $N=3$. (D)

(D) Left, microscopic images of Hoechst-stained EV (CaPJ170) and $C S A 6^{O E}$ strain (CaPJ176) after $8 \mathrm{~h}$ of growth under indicated conditions of Dox $(50 \mu \mathrm{g} / \mathrm{ml})$. BF, bright-field. Scale bar, $10 \mu \mathrm{m}$. Right, quantitation of different cell types at the indicated timepoints; $n \geq 100$ cells. (E) Top, representative micrographs of spindle morphology in the largebudded cells of EV (CaPJ172) and $C S A 6^{O E}$ strain $(\mathrm{CaPJ} 178)$ after $8 \mathrm{~h}$ of growth under indicated conditions of Dox $(50 \mu \mathrm{g} / \mathrm{ml})$. SPBs and MTs are marked by Tub4-GFP and Tub1-mCherry, respectively. Scale bar, $1 \mu \mathrm{m}$. Bottom, the proportion of the large-budded cells with indicated SPB phenotypes; $n \geq 100$ cells. 
A

B

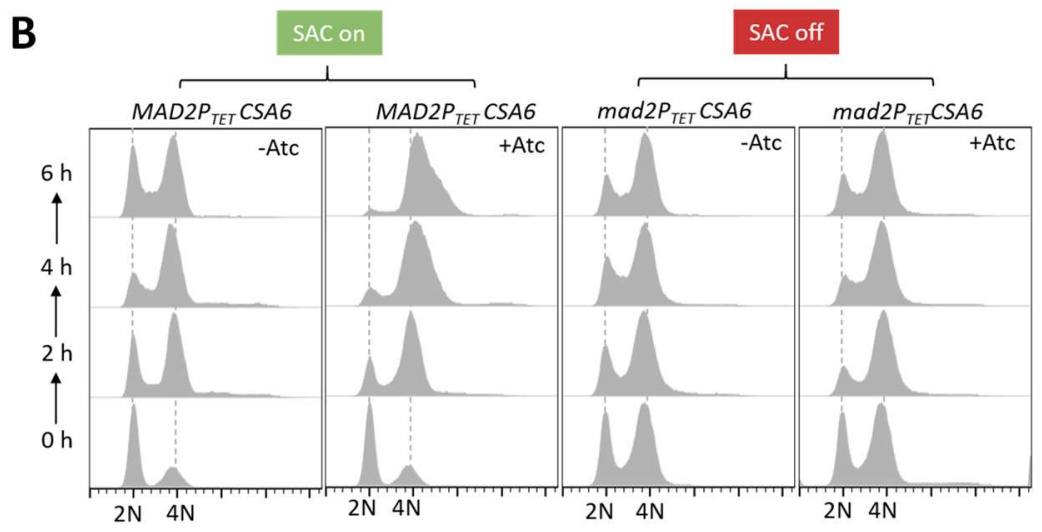

C
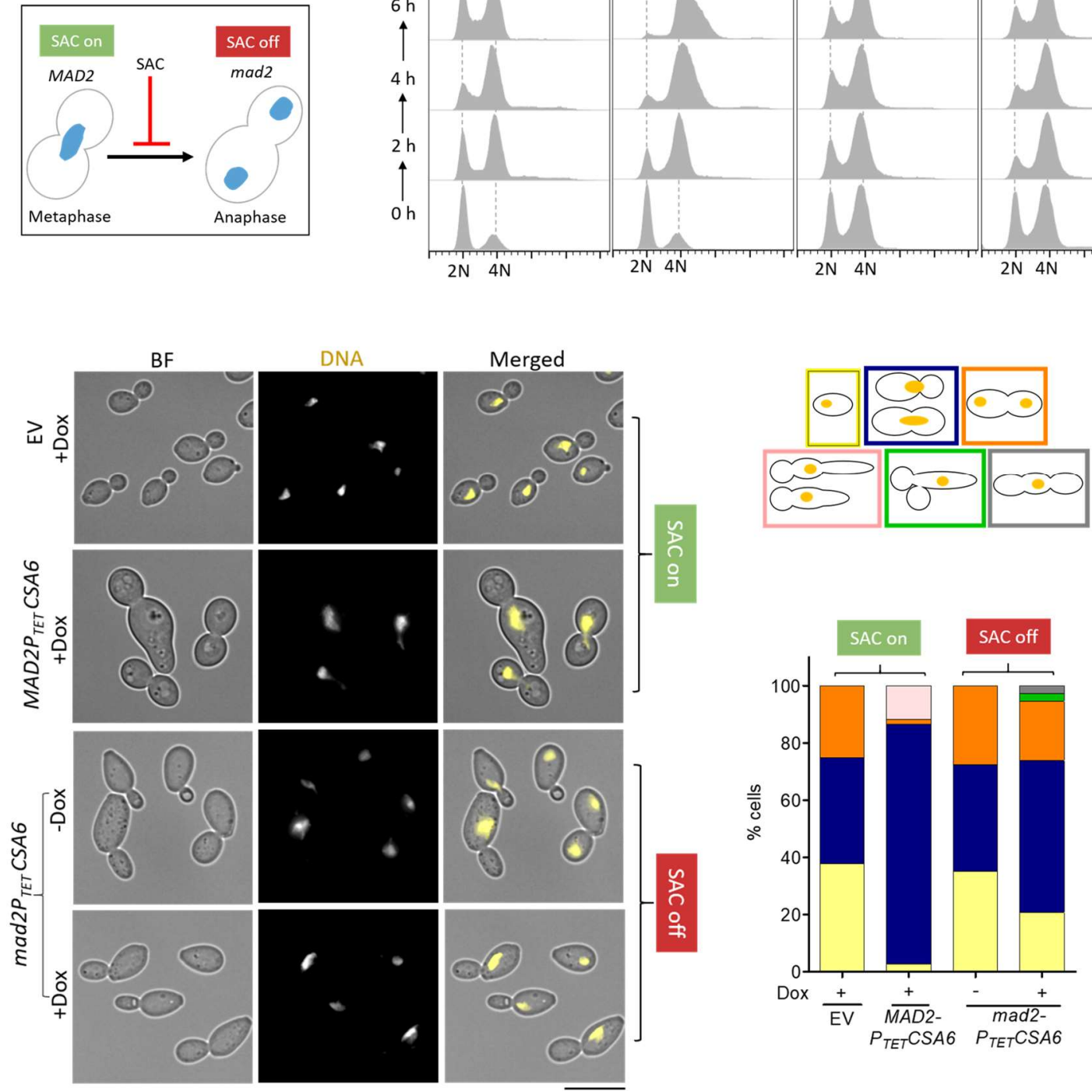

Fig. 4. The G2/M cell cycle arrest in the $\mathrm{CSA6}^{\mathrm{OE}}$ mutant is mediated by Mad2. (A) The G2/M arrest posed by SAC in response to an improper chromosome-spindle attachment is relieved in the absence of Mad2, allowing cells to transit from metaphase to anaphase. (B) Flow cytometric DNA content analysis in CaPJ176 (MAD2CSA6 $\left.{ }^{O E}\right)$ and CaPJ197 (mad2CSA6 $\left.{ }^{O E}\right)$ at the indicated times, in presence or absence of Atc $(3 \mu \mathrm{g} / \mathrm{ml}) ; N=3$. (C) Left, microscopic images of CaPJ170 (EV), CaPJ176 (MAD2CSA6 ${ }^{O E}$ ) and CaPJ197 (mad2CSA6 ${ }^{\circ E}$ ) following Hoechst staining, after $8 \mathrm{~h}$ of growth under indicated conditions of Dox $(50 \mu \mathrm{g} / \mathrm{ml})$. Scale bar, $10 \mu \mathrm{m}$. Right, quantitation of the indicated cell types; $n \geq 100$ cells. 
A

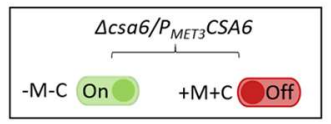

B

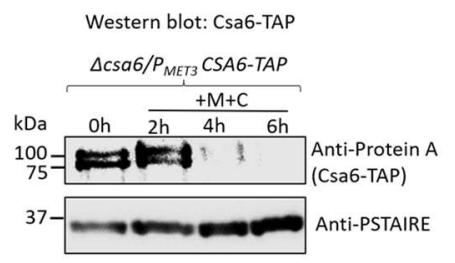

D

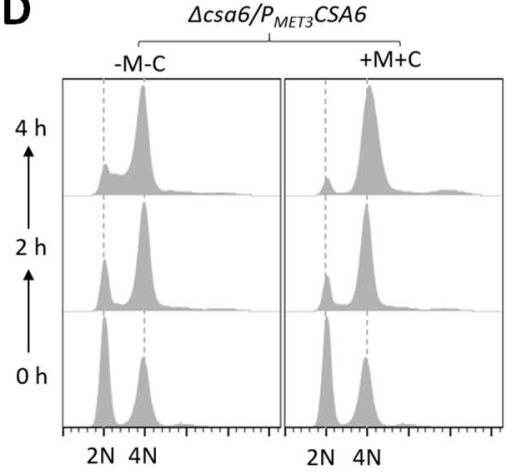

C
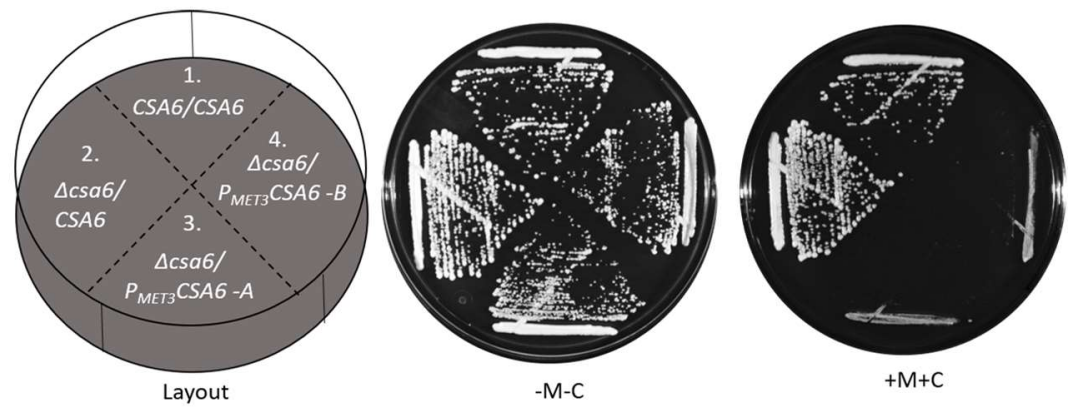

E
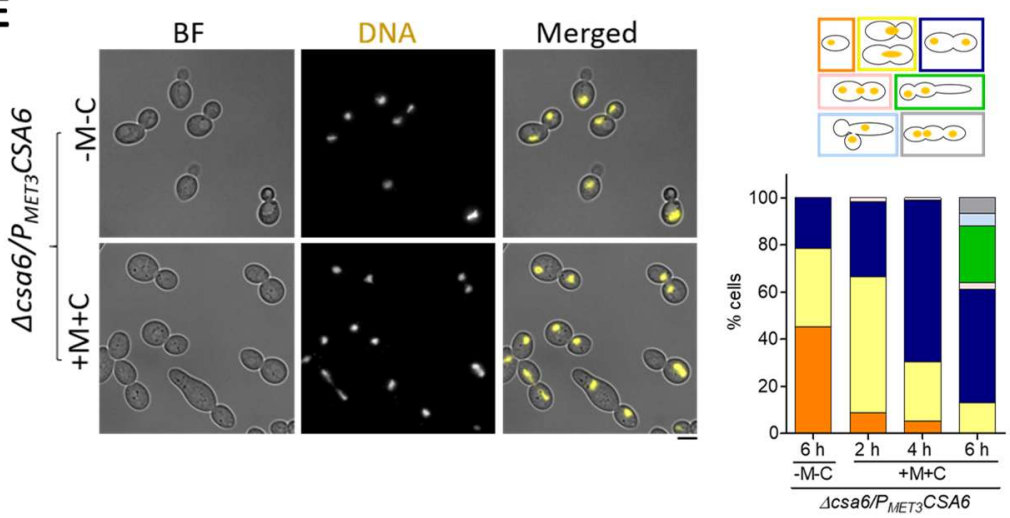

F
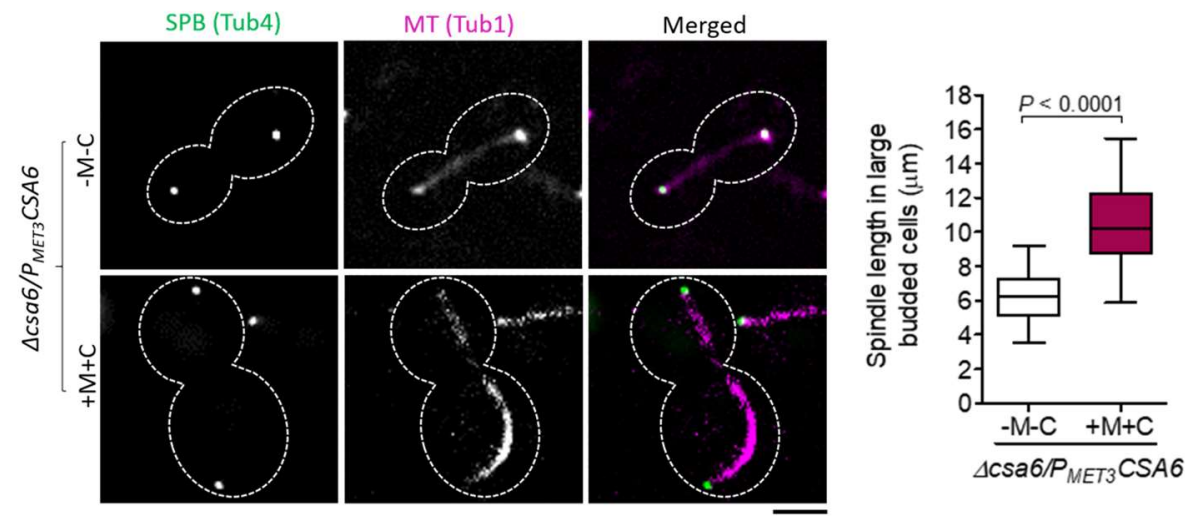

Fig. 5. Csa6 depletion causes late anaphase/telophase arrest with a hyper-extended mitotic spindle in C. albicans. (A) The MET3 promoter system for depleting cellular levels of Csa6. The MET3 promoter can be conditionally repressed in presence of methionine (Met/M) and cysteine (Cys/C). (B) (B) Western blot analysis using anti-Protein A antibodies revealed time dependent depletion of Csa6-TAP in $C S A 6^{P S D}$ strain (CaPJ212), grown under repressive conditions (YPDU $+5 \mathrm{mM}$ Met and $5 \mathrm{mM}$ Cys) for indicated time interval; $N=2$. (C) Csa6 is essential for viability in C. albicans. Strains with indicated genotypes, (1) SN148, (2) CaPJ209, (3 and 4) CaPJ210 (two transformants) were streaked on agar plates with permissive (YPDU-Met-Cys) or repressive 
1205 (YPDU $+5 \mathrm{mM}$ Met and $5 \mathrm{mM}$ Cys) media and incubated at $30^{\circ} \mathrm{C}$ for two days. (D) Cell cycle 1206 analysis of CaPJ210 (CSA6 $\left.{ }^{P S D}\right)$ by flow cytometry under permissive (YPDU-Met-Cys) and 1207 repressive conditions (YPDU $+5 \mathrm{mM}$ Met and $5 \mathrm{mM}$ Cys) at the indicated time intervals; $N=3$.

1208 (E) Left, microscopic images of Hoechst stained CaPJ210 $\left(C S A 6^{P S D}\right)$ cells grown under 1209 permissive (YPDU-Met-Cys) or repressive (YPDU $+5 \mathrm{mM}$ Met and $5 \mathrm{mM}$ Cys) conditions for 6 1210 h. BF bright-field. Scale bar, $5 \mu \mathrm{m}$. Right, quantitation of different cell types at the indicated time1211 points; $n \geq 100$ cells. (F) Left, micrograph showing Tub4-GFP and Tub1-mCherry (representing 1212 mitotic spindle) in the large-budded cells of CaPJ211 (CSA $\left.6^{P S D}\right)$ after $6 \mathrm{~h}$ of growth under 1213 permissive (YPDU-Met-Cys) or repressive (YPDU + $5 \mathrm{mM}$ Met and $5 \mathrm{mM}$ Cys) conditions.

1214 Scale bar, $3 \mu \mathrm{m}$. Right, quantitation of the distance between the two SPBs, along the length of the 1215 MT (representing spindle length), in large-budded cells of CaPJ211 (CSA6 $\left.6^{P S D}\right)$ under permissive $1216 \quad(n=32)$ or repressive $(n=52)$ conditions. Paired $t$-test, one-tailed, $P$-value shows a significant 1217 difference. 
Figure 6

A

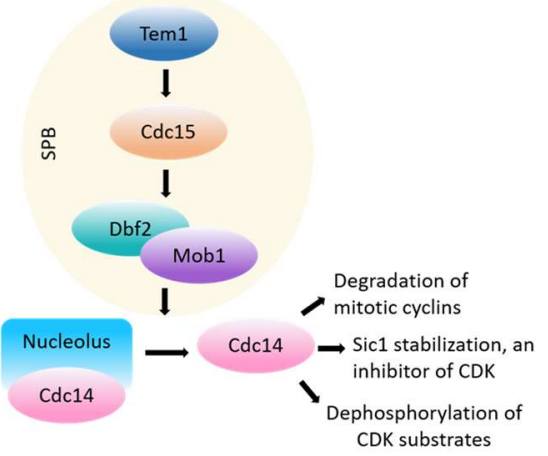

D

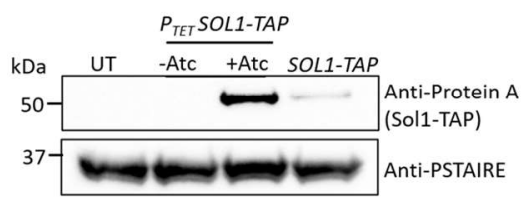

B

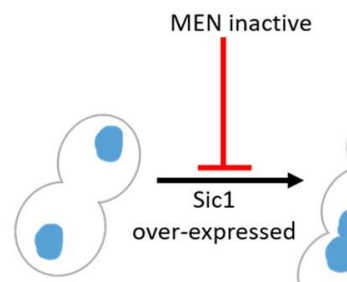

Late anaphase/ telophase arrest
C

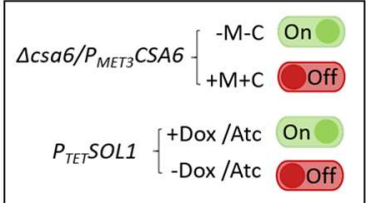

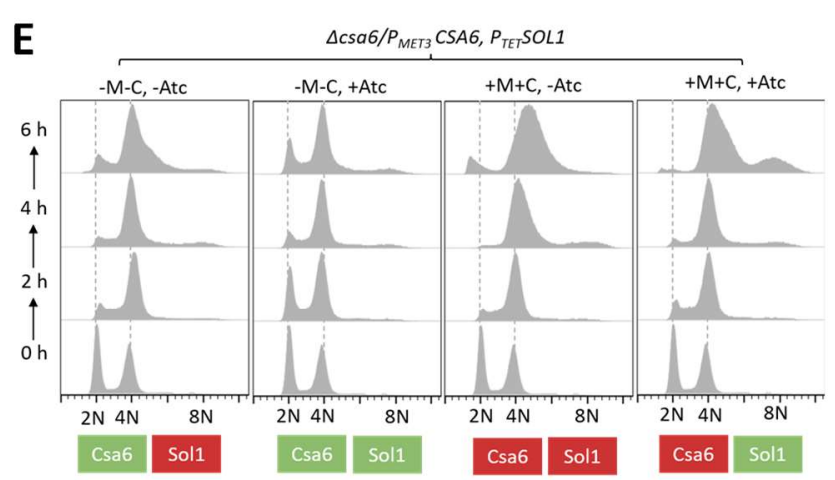

\section{$\mathbf{F}$}
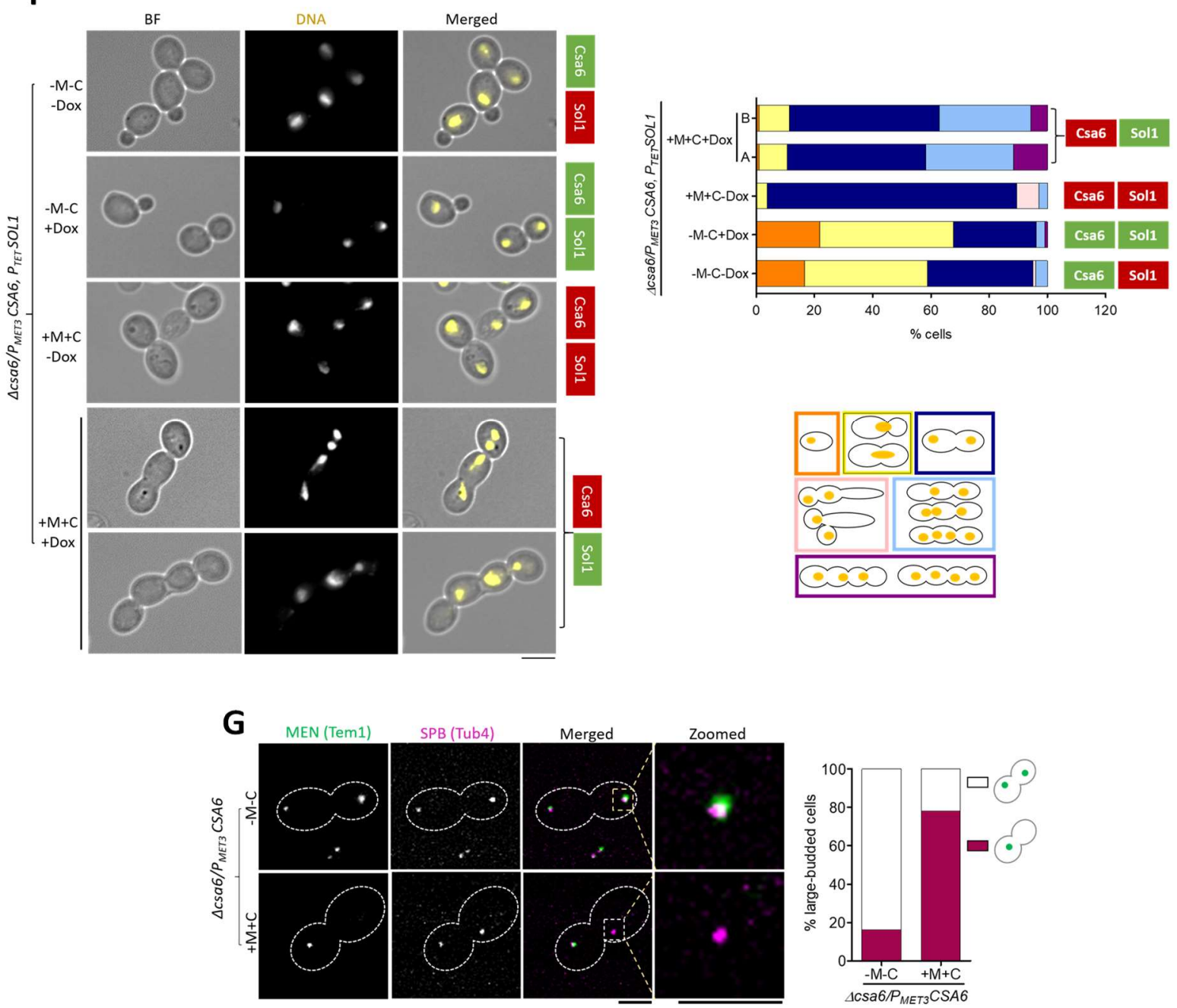
Fig. 6. Csa6 is required for mitotic exit in $C$. albicans. (A) The MEN components in $S$. cerevisiae. At SPB, Nud1 acts as a scaffold. The ultimate target of the MEN is to activate Cdc14 phosphatase, which remains entrapped in the nucleolus in an inactive state until anaphase. Cdc14 release brings about mitotic exit and cytokinesis by promoting degradation of mitotic cyclins, inactivation of mitotic CDKs through Sic1 accumulation and dephosphorylation of the CDK substrates ${ }^{64}$. (B) Inhibition of the MEN signaling prevents cells from exiting mitosis and arrests them at late anaphase/telophase. Bypass of cell cycle arrest due to the inactive MEN, viz. by overexpression of Sic1-a CDK inhibitor, results in the chain of cells with multiple nuclei ${ }^{98,99}$. (C) A combination of two regulatable promoters, $\mathrm{P}_{\text {TET }}$ and $\mathrm{P}_{\text {MET3 }}$, was used to overexpress $C$. albicans homolog of Sic1, called SOL1 ( $\underline{\text { Sic }}$ one-like), in Csa6-depleted cells. The resulting strain, CaPJ215, can be conditionally induced for both SOL1 overexpression upon Atc/Dox addition and Csa6 depletion upon Met (M)/Cys (C) addition. (D) Protein A western blot analysis showed increased levels of Sol1 (TAP-tagged) in the SOL1 ${ }^{O E}$ mutant (CaP217, P PETSOL1-TAP) after $6 \mathrm{~h}$ induction in presence of Atc $(3 \mu \mathrm{g} / \mathrm{ml})$ in comparison to the uninduced culture (-Atc) or SOL1 expression from its native promoter (CaPJ216, SOL1-TAP); $N=2$. PSTAIRE was used as a loading control. UT, untagged control (SN148). (E) Flow cytometric analysis of cell cycle progression in CaPJ215 at indicated time intervals under various growth conditions, as indicated; $N=3$. Dox: $50 \mu \mathrm{g} / \mathrm{ml}$, Met: $5 \mathrm{mM}$, Cys: $5 \mathrm{mM}$. (F) Left, Hoechst staining of CaPJ215 after 6 h of growth under indicated conditions of Dox $(50 \mu \mathrm{g} / \mathrm{ml})$, Met $(5 \mathrm{mM})$ and Cys $(5 \mathrm{mM}) ; n \geq 100$ cells. BF bright-field. Scale bar, $5 \mu \mathrm{m}$. Right, percent distribution of the indicated cell phenotypes; $n$ $\geq 100$ cells. (G) Left, co-localization analysis of Tem1-GFP and Tub4-mCherry in large-budded cells of CaPJ218 (CSA6 ${ }^{P S D}$ ) under permissive (YPDU-Met-Cys) or repressive conditions (YPDU $+5 \mathrm{mM}$ Met and $5 \mathrm{mM}$ Cys). Scale bar, $3 \mu \mathrm{m}$. Right, the proportion of the large-budded cells with indicated Tem 1 phenotypes; $n \geq 100$ cells. 
A

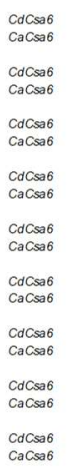

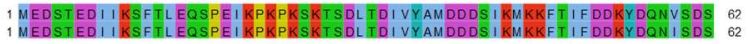

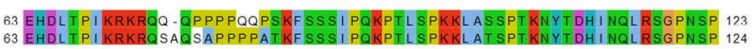

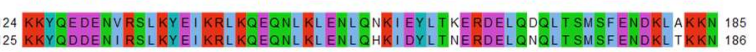

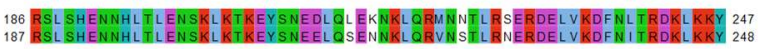

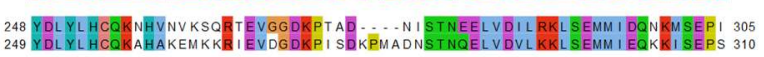

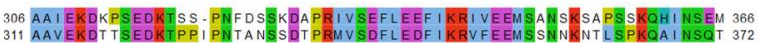

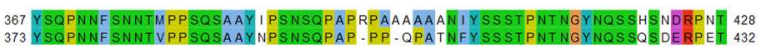

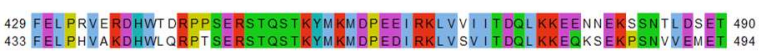

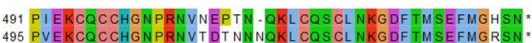

B

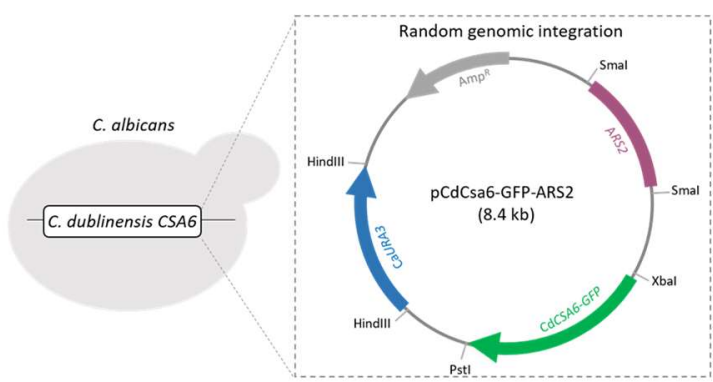

C

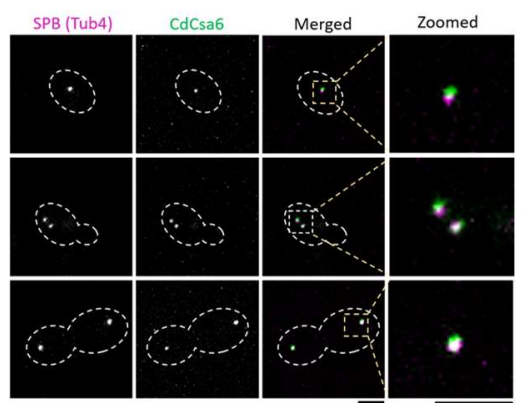

D
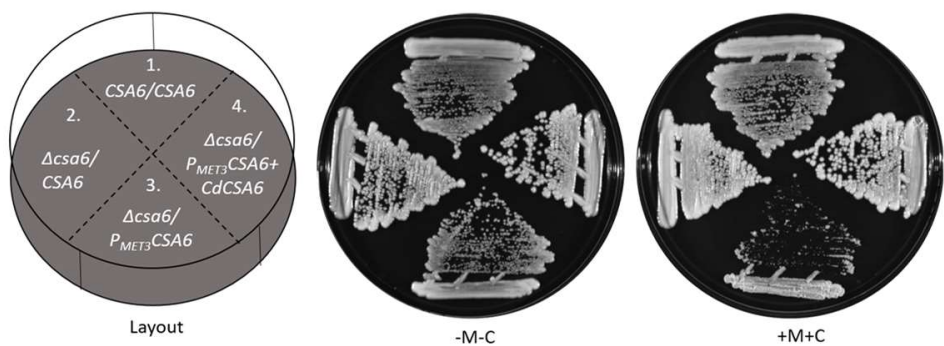

Fig. 7. Ectopic expression and functional conservation of CdCsa6 in C. albicans. (A) Pairwise alignment of amino acid sequences of Csa6 proteins in C. albicans (CaCsa6) and $C$. dubliniensis (CdCsa6) by Clustal Omega, visualized using Jalview. (B) Ectopic expression of CdCsa6 in C. albicans by random genomic integration of the ARS-containing plasmid. Vector map of pCdCsa6-GFP-ARS2 depicts the cloned sites of $\mathrm{CaURA3}, \mathrm{Ca} A R S 2$ and CdCSA6-GFP. The CdCSA6-GFP fragment contains the GFP tag, CdCSA6 (ORF Cd36_16290) without the stop codon and the promoter region of CdCSA6. (C) CdCsa6 localizes to the SPB. Representative micrographs showing CdCsa6GFP localization at different cell cycle stages in CaPJ300. Tub4mCherry was used as an SPB marker. Scale bar, $3 \mu \mathrm{m}$. (D) CdCsa6 functionally complements CaCsa6. Strains with indicated genotypes, (1) SN148, (2) CaPJ300, (3) CaPJ301 and (4) CaPJ302, were streaked on agar plates with permissive (YPDU-Met-Cys) or repressive (YPDU $+5 \mathrm{mM}$ Met and $5 \mathrm{mM} \mathrm{Cys}$ ) media and incubated at $30^{\circ} \mathrm{C}$ for two days. 
A

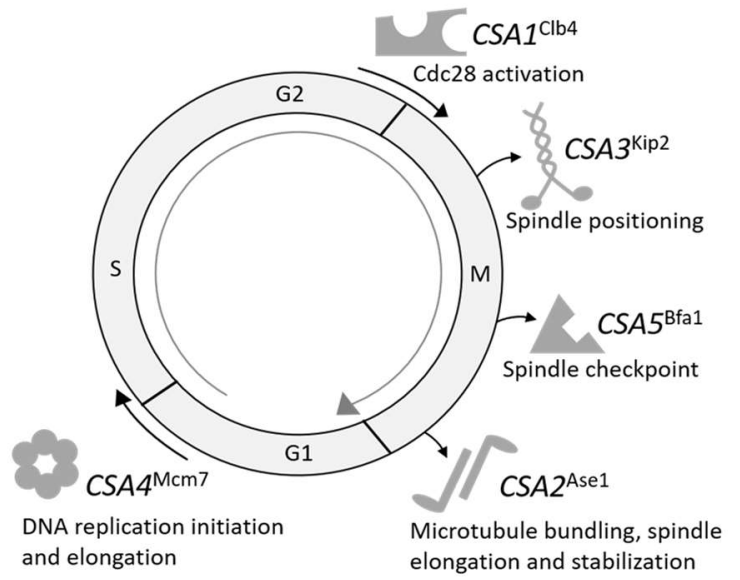

B

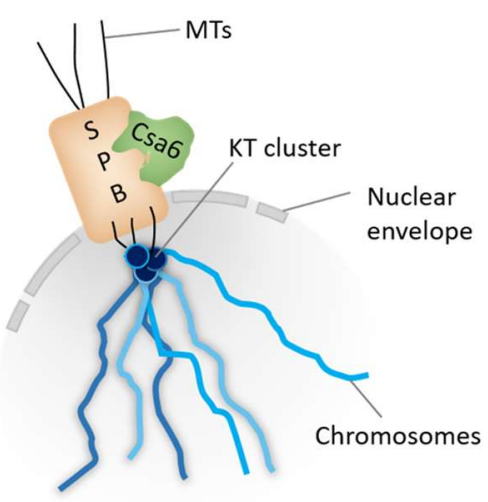

C

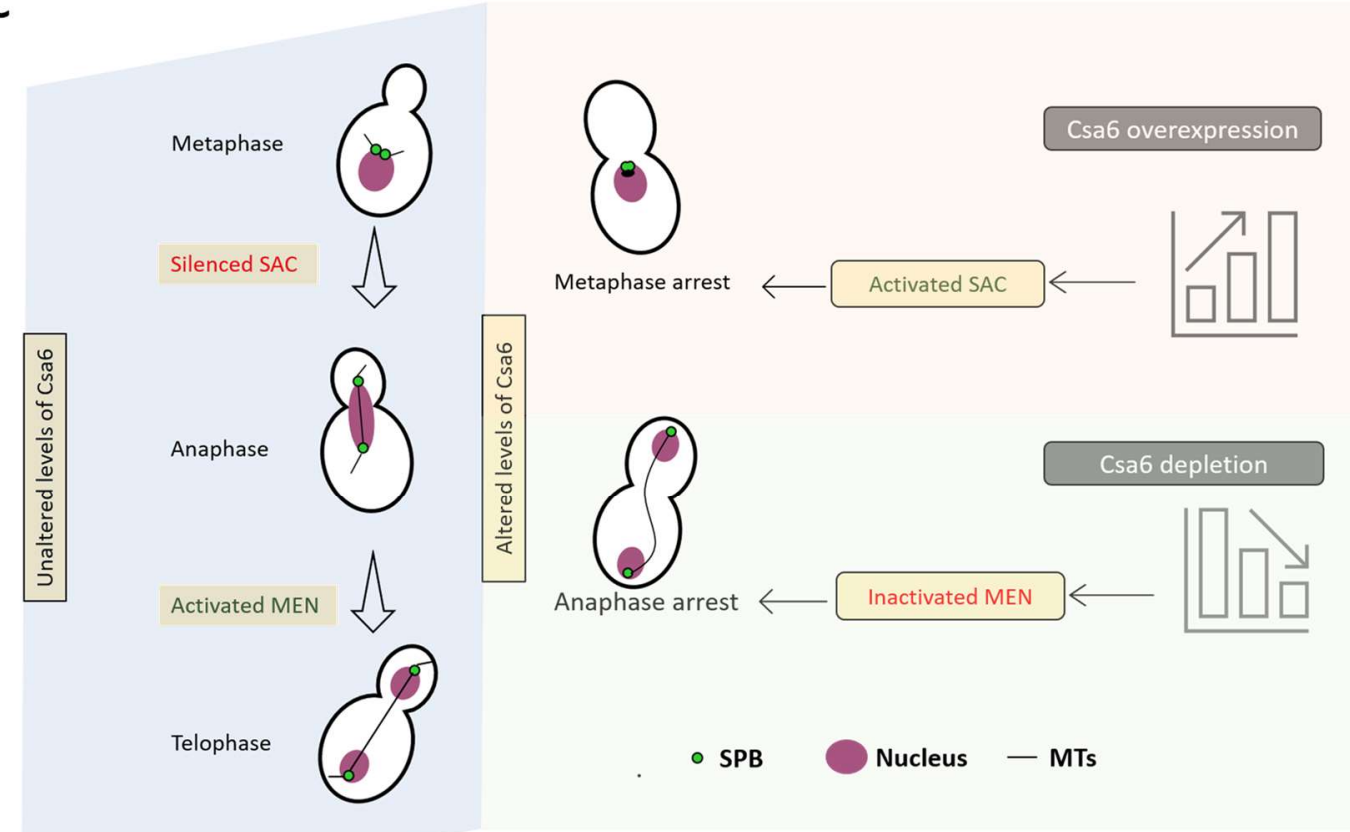

Fig. 8. Csa6 levels are fine-tuned at various stages of the cell cycle to ensure both mitotic

progression and mitotic exit in $C$. albicans. (A) A diagram illustrating the functions of the

identified CSA genes except CSA6 in various phases and phase transitions of the cell cycle. (B)

Schematic depicting the approximate position of Csa6 with respect to SPB and KT. In $C$.

albicans, SPBs and clustered KTs remain in close proximity throughout the cell cycle, while Csa6

remains constitutively localized to the SPBs. (C) A model summarizing the effects of

overexpression or depletion of Csa6 in C. albicans. A wild-type cell with unperturbed Csa6 levels

progresses through the mitotic cell cycle. Overexpression of CSA6 alters the mitotic spindle 
1306 dynamics which might lead to improper KT-MT attachments, prompting SAC activation and 1307 G2/M arrest. In contrast, decreased levels of Csa6 inhibit the MEN signaling pathway, probably 1308 by affecting Tem1 recruitment to the SPBs, resulting in cell cycle arrest at the anaphase stage. 
Table 1. Overexpression phenotypes of $C S A$ genes in $C$. albicans and $S$. cerevisiae

\begin{tabular}{|c|c|c|c|c|c|}
\hline $\begin{array}{l}C S A \\
\text { gene }\end{array}$ & $\begin{array}{c}\text { C. albicans } \\
\text { ORF no. }\end{array}$ & $\begin{array}{c}S . \\
\text { cerevisiae } \\
\text { homolog }\end{array}$ & $\begin{array}{c}\text { Overexpression } \\
\text { phenotype }(C . \\
\text { albicans) }\end{array}$ & $\begin{array}{c}\text { Overexpression } \\
\text { phenotype ( } S . \\
\text { cerevisiae) }\end{array}$ & Reference \\
\hline CSA1 & 19.7186 & CLB4 & $\begin{array}{l}\text { Increased CIN } \\
\text { involving non-CL } \\
\text { events }\end{array}$ & $\begin{array}{l}\text { Shift towards } 2 \mathrm{~N} \\
\text { (diploid) DNA content }\end{array}$ & 100 \\
\hline$C S A 2$ & 19.7377 & ASE 1 & $\begin{array}{l}\text { Increased CIN } \\
\text { involving non-CL } \\
\text { events }\end{array}$ & $\begin{array}{l}\text { i) CIN involving loss } \\
\text { of an artificial } \\
\text { chromosome fragment } \\
\text { or rearrangements/ } \\
\text { gene conversion } \\
\text { events. } \\
\text { ii) Spindle checkpoint } \\
\text { dependent delay in } \\
\text { entering anaphase } \\
\text { upon HU treatment }\end{array}$ & 14,75 \\
\hline$C S A 3$ & 19.1747 & KIP2 & $\begin{array}{l}\text { Increased CIN } \\
\text { involving non-CL } \\
\text { events }\end{array}$ & $\begin{array}{l}\text { Shift towards } 2 \mathrm{~N} \\
\text { (diploid) DNA content }\end{array}$ & 81,100 \\
\hline CSA4 & 19.202 & $M C M 7$ & $\begin{array}{l}\text { Shift towards } 4 \mathrm{~N} \\
\text { (diploid) DNA } \\
\text { content, G2/M } \\
\text { arrest }\end{array}$ & NA & NA \\
\hline CSA5 & 19.608 & $B F A 1$ & $\begin{array}{l}\text { Shift towards 4N } \\
\text { (diploid) DNA } \\
\text { content, anaphase } \\
\text { arrest }\end{array}$ & $\begin{array}{l}\text { Shift towards } 2 \mathrm{~N} \\
\text { (diploid) DNA content, } \\
\text { Anaphase arrest }\end{array}$ & 101 \\
\hline CSA6 & 19.1447 & NA & $\begin{array}{l}\text { Shift towards 4N } \\
\text { (diploid) DNA } \\
\text { content, G2/M } \\
\text { arrest }\end{array}$ & NA & NA \\
\hline
\end{tabular}




\section{Supplementary Files}

This is a list of supplementary files associated with this preprint. Click to download.

- Dataset1.xlsx

- Jaitlyetal.supplenaturecom.pdf 